\title{
BLACK HOLE FORMATION IN FAILING CORE-COLLAPSE SUPERNOVAE
}

\author{
Evan O'Connor and Christian D. OtT \\ TAPIR, Mailcode 350-17, California Institute of Technology, Pasadena, CA 91125, USA; evanoc@ tapir.caltech.edu, cott@ tapir.caltech.edu \\ Received 2010 October 26; accepted 2011 January 10; published 2011 March 7
}

\begin{abstract}
We present results of a systematic study of failing core-collapse supernovae and the formation of stellar-mass black holes (BHs). Using our open-source general-relativistic 1.5D code GR1D equipped with a three-species neutrino leakage/heating scheme and over 100 presupernova models, we study the effects of the choice of nuclear equation of state (EOS), zero-age main sequence (ZAMS) mass and metallicity, rotation, and mass-loss prescription on BH formation. We find that the outcome, for a given EOS, can be estimated, to first order, by a single parameter, the compactness of the stellar core at bounce. By comparing protoneutron star (PNS) structure at the onset of gravitational instability with solutions of the Tolman-Oppenheimer-Volkof equations, we find that thermal pressure support in the outer PNS core is responsible for raising the maximum PNS mass by up to 25\% above the cold NS value. By artificially increasing neutrino heating, we find the critical neutrino heating efficiency required for exploding a given progenitor structure and connect these findings with ZAMS conditions, establishing, albeit approximately, for the first time based on actual collapse simulations, the mapping between ZAMS parameters and the outcome of core collapse. We also study the effect of progenitor rotation and find that the dimensionless spin of nascent BHs may be robustly limited below $a^{*}=J c / G M^{2}=1$ by the appearance of nonaxisymmetric rotational instabilities.
\end{abstract}

Key words: black hole physics - equation of state - hydrodynamics - neutrinos - stars: evolution - stars: mass-loss - stars: neutron - supernovae: general

Online-only material: color figures

\section{INTRODUCTION}

Massive stars with zero-age main sequence (ZAMS) masses $M_{\text {ZAMS }}$ in the range of $8-10 M_{\odot} \lesssim M_{\text {ZAMS }} \lesssim 100-150 M_{\odot}$ end their lives with the gravitationally induced catastrophic collapse of their electron-degenerate iron core to nuclear densities. There, the nuclear equation of state (EOS) stiffens and stabilizes the inner core, which overshoots its new equilibrium and bounces back, launching a hydrodynamic shock. The shock initially races through the still collapsing outer core, but soon stalls and turns into an accretion shock (at $r \sim 100-200 \mathrm{~km}$ ) due to the dissociation of heavy nuclei at the shock front and neutrino losses from the postshock region (Bethe 1990). The shock must be revived to drive a core-collapse supernova (CCSN) and the precise nature of the responsible CCSN mechanism has been a topic of intense research for decades (e.g., Arnett 1966; Colgate \& White 1966; Bethe \& Wilson 1985; Janka et al. 2007; Burrows et al. 2006, 2007b; Murphy \& Burrows 2008; Marek \& Janka 2009; Nordhaus et al. 2010, and references there in).

A neutron star (NS) is left behind by a CCSN that explodes soon after bounce and successfully unbinds its stellar mantle. However, a stellar-mass black hole (BH) may be the outcome: (1) if in a successful, but perhaps weak, CCSN fallback accretion pushes the nascent NS over its mass limit; (2) if nuclear phase transitions during protoneutron star (PNS) cooling occur or if PNS cooling reduces pressure support in a hyper-massive PNS; or (3) if the CCSN mechanism lacks efficacy and fails to revive the shock and continued accretion pushes the PNS over its maximum mass. In this last channel to a stellar-mass BH, there is no electromagnetic (EM) signal other than the disappearance of the original star. Such "unnovae" (Kochanek et al. 2008), failing CCSNe, are the topic of this paper.

In ordinary massive stars that hydrostatically form degenerate iron cores, BH formation, in any scenario, is never prompt (e.g.,
Burrows 1988; Ott \& O'Connor 2010). It is always preceded by an extended PNS phase giving rise to copious emission of both neutrinos (Burrows 1988; Beacom et al. 2001) and gravitational waves (Ott 2009) until the PNS is engulfed by the BH horizon. The EM silence expected in a failed CCSN may be broken after all, if sufficient and appropriately distributed angular momentum is present to allow for a Keplerian accretion disk to form near the BH, permitting a collapsar (Woosley 1993) gammaray burst (GRB) central engine to operate and drive relativistic outflows.

It is currently unclear what fraction of massive stars form $\mathrm{BHs}$ and through which channel. Pre-explosion observations of progenitors of successful CCSNe suggest progenitor masses $\lesssim 17-20 M_{\odot}$ (Smartt et al. 2009) for standard Type II-P supernovae. Assuming, as suggested by Smartt et al. (2009), that most other CCSNe fail or make BHs after a successful explosion, this would correspond to a BH fraction of $\lesssim 30 \%-35 \%$ of massive stars above $8 M_{\odot}$. However, alternative interpretations exist and have been summarized by Smith et al. (2010). Theoretical work by Timmes et al. (1996), Fryer (1999), Heger et al. (2003), and Eldridge \& Tout (2004) provided rough estimates on the outcomes of stellar collapse as a function of progenitor ZAMS mass and metallicity. Leaving effects due to binary evolution aside, Zhang et al. (2008) performed an extensive study of fallback in artificially driven spherically symmetric CCSN explosions and estimated that zero-metallicity stars form BHs in $20 \%-50 \%$ of all core-collapse events with an average $\mathrm{BH}$ mass of $6-10 M_{\odot}$. For solar-metallicity stars, due to increased mass loss during evolution, Zhang et al. (2008) found BHs to form at a significantly lower rate and initial mass. They predict $\mathrm{BH}$ fractions in the range of $10 \%-25 \%$ with typical initial $\mathrm{BH}$ masses of $3 M_{\odot}$. This is in rough agreement with previous population synthesis calculations of Fryer \& Kalogera (2001) and Belczynski et al. (2002). 
Early spherically symmetric (one-dimensional, 1D) simulations of $\mathrm{BH}$ formation in failing CCSNe were carried out by Wilson (1971) and van Riper \& Arnett (1978). Burrows (1988) performed a set of quasi-stationary 1D PNS accretion and cooling simulations to investigate the possibility of $\mathrm{BH}$ formation in SN 1987A. Delayed BH formation (by tens of seconds), due to, e.g., a nuclear EOS phase transition, was studied by Baumgarte et al. (1996a, 1996b). More recently, 1D full Boltzmann neutrino radiation-hydrodynamics calculations of failing nonrotating CCSNe were carried out by Liebendörfer et al. (2004) and more recently Sumiyoshi et al. (2007, 2008, 2009) and Fischer et al. (2009). These studies provided detailed neutrino signature predictions for $\mathrm{BH}$-forming core-collapse events. However, owing to the complexity and computational expense of such Boltzmann-transport calculations, these groups could consider only very limited sets of progenitor models and EOS. Simplified axisymmetric (2D) simulations of $\mathrm{BH}$ formation in rotating core collapse were first performed in a series of papers by Sekiguchi \& Shibata $(2004,2005)$ and Shibata \& Sekiguchi (2005). These authors used simplified EOS, no neutrino treatment, and artificially constructed initial conditions and found prompt $\mathrm{BH}$ formation. Recently, the same authors performed a small set of 2D simulations with a finite-temperature nuclear EOS and a leakage scheme for neutrinos (Sekiguchi \& Shibata 2010) and considered collapse, BH formation, and subsequent evolution in an artificially constructed progenitor with an iron core mass of $\sim 13 M_{\odot}$ and constant specific entropy of $8 k_{B} /$ baryon, initial conditions that are inconsistent with those at the precollapse stage of CCSN progenitors.

In this paper, our focus is on studying and establishing the systematics of failing $\mathrm{CCSNe}$ and $\mathrm{BH}$ formation. For this, we employ the spherically symmetric general-relativistic (GR) open-source code GR1D (O'Connor \& Ott 2010) that can handle rotation in an approximate angle-averaged way ("1.5D") and sacrifice accuracy in the neutrino treatment by employing an efficient energy-averaged three-species neutrino leakage/ heating scheme instead of full transport. The efficiency of GR1D enables us to perform more than $\sim 700$ collapse calculations, investigating for the first time in detail the effects of variations in nuclear EOS, progenitor ZAMS mass and metallicity, neutrino heating efficiency, and precollapse rotational configuration. We employ four different finite-temperature nuclear EOS and draw a total of 106 progenitor models from six stellar evolution studies.

In Section 2, we review the features of our 1.5D GR hydrodynamics code GR1D, discuss our neutrino leakage/heating scheme, and introduce the set of employed EOS. Section 3 introduces our progenitor model set, numerical grid setup, and precollapse rotational setup. In Section 4.1, we introduce key aspects of failing CCSNe and $\mathrm{BH}$ formation by discussing the evolution of BH formation in a fiducial nonrotating $40 M_{\odot}$ solarmetallicity progenitor. We go on in Section 4.3 to study the influence of the EOS and thermal effects on the time to $\mathrm{BH}$ formation and on the maximum (baryonic and gravitational) mass of the PNS. We discover that for nuclear EOS with physically plausible stiffness, the maximum (baryonic and gravitational) mass of the PNS is always greater than the corresponding cold NS mass and discuss that the difference is due entirely to thermal pressure support of material in the hot outer PNS core. This effect is strongest for the softest considered EOS and decreases with increasing EOS stiffness. In Section 4.4, we analyze the impact of variations in progenitor structure on the time to $\mathrm{BH}$ formation and the maximum PNS mass in failing CCSNe. We find that the postbounce dynamics can be predicted rather robustly by a single parameter, the compactness of the progenitor structure at core bounce. The same approximate single-parameter dependence emerges in Section 4.5, where we determine the neutrino heating efficiencies required (modulo ignored multidimensional effects) to induce a neutrino-driven explosion in a large set of progenitors. The combined results of Sections 4.4 and 4.5 allow us to make predictions on the outcome of core collapse for progenitors with varying ZAMS mass and metallicity in Section 4.6. As we discuss in that section, mass loss may be the greatest uncertainty in connecting ZAMS parameters to core-collapse results. In Section 4.7, we present results from the first rotating $\mathrm{BH}$ formation simulations in the CCSN context. Varying the precollapse rotation rate in Section 4.7.1, we find that, not unexpectedly, increased rotation leads to a delay of $\mathrm{BH}$ formation and greater maximum PNS masses. We also observe that the birth spin of Kerr BHs in nature appears to be robustly limited to values below $a^{\star}=J / M^{2} \lesssim 0.9$ by the likely appearance of nonaxisymmetric rotational instabilities that redistribute or radiate angular momentum. This finding requires confirmation by 3D simulations. We go on in Section 4.7.2 to discuss the collapse evolution of a set of progenitors that were evolved from the ZAMS with a $1.5 \mathrm{D}$ treatment of rotation and discuss their viability as collapsar-type long-GRB progenitors. Finally, in Section 5, we critically summarize our work and conclude.

\section{METHODS}

\subsection{GR Hydrodynamics}

GR1D (O'Connor \& Ott 2010) is a spherically symmetric GR hydrodynamics code developed for the study of stellar collapse and BH formation. It is available for download at http://stellarcollapse.org. GR1D, based on the previous work of Gourgoulhon (1991) and Romero et al. (1996), is Eulerian and uses the radial gauge_-polar slicing coordinates that have the simplifying property of a vanishing shift vector. Here, we briefly outline the basics of the curvature and hydrodynamics equations and refer the reader to O'Connor \& Ott (2010) for full details and derivation. We assume spacelike signature $(-,+,+,+)$ and, unless noted otherwise, use units of $G=c=M_{\odot}=1$. The metric of GR1D is given by the line element

$$
d s^{2}=-\alpha(r, t)^{2} d t^{2}+X(r, t)^{2} d r^{2}+r^{2} d \Omega^{2},
$$

where $\alpha(r, t)=\exp (\Phi(r, t))$ with $\Phi(r, t)$ being the metric potential. $X(r, t)=[1-2 m(r) / r]^{-1 / 2}$, where $m(r)$ is the enclosed gravitational mass. We assume an ideal fluid with stress energy given by

$$
T^{\mu \nu}=\rho h u^{\mu} u^{\nu}+g^{\mu v} P,
$$

where $\rho$ is the matter density, $P$ is the fluid pressure and $h=1+\epsilon+P / \rho$ is the specific enthalpy with $\epsilon$ being the specific internal energy. $u^{\mu}$ in Equation (2) is the 4-velocity of the fluid, and without rotation, taken to be $u^{\mu}=\left(W / \alpha, W v^{r}, 0,0\right)$, where $W=\left[1-v^{2}\right]^{-1 / 2}$ is the Lorentz factor and $v=X v^{r}$ is the physical velocity. For a given matter configuration, the Hamiltonian and momentum constraint equations give differential equations for both $m(r)$ and $\Phi(r)$,

$$
\begin{aligned}
m(r)= & 4 \pi \int_{0}^{r}\left(\rho h W^{2}-P+\tau_{m}^{v}\right) r^{\prime 2} d r^{\prime} \\
\Phi(r, t)= & \int_{0}^{r} X^{2}\left[\frac{m\left(r^{\prime}, t\right)}{r^{\prime 2}}+4 \pi r^{\prime}\left(\rho h X^{2} u^{r^{\prime}} u^{r^{\prime}}+P+\tau_{\Phi}^{v}\right)\right] d r^{\prime} \\
& +\Phi_{0},
\end{aligned}
$$


where $\Phi_{0}$ is determined by matching the metric at the star's surface to the Schwarzschild metric. The neutrino terms, $\tau_{m}^{\nu}$ and $\tau_{\Phi}^{\nu}$, account for trapped neutrinos and their detailed form is given in O'Connor \& Ott (2010). We obtain the fluid evolution equations by expanding the local fluid rest-frame conservation laws, $\nabla_{\mu} T^{\mu \nu}=0$ and $\nabla_{\mu} J^{\mu}=0$, in the coordinates of GR1D. The conservation laws become

$$
\partial_{t}(\vec{U})+\frac{1}{r^{2}} \partial_{r}\left(\frac{\alpha r^{2}}{X} \vec{F}\right)=\overrightarrow{\mathcal{S}}
$$

where $\vec{U}=\left(D, D Y_{e}, S^{r}, \tau\right)$ are the conserved variables, given in terms of the primitive fluid variables $\rho, Y_{e}, \epsilon, P$, and $v$ as

$$
\vec{U}=\left(\begin{array}{c}
D \\
D Y_{e} \\
S^{r} \\
\tau
\end{array}\right)=\left(\begin{array}{c}
X \rho W \\
X \rho W Y_{e} \\
\rho h W^{2} v \\
\rho h W^{2}-P-D
\end{array}\right)
$$

The spatial fluxes in Equation (5) are given by

$$
\vec{F}=\left(D v, D Y_{e} v, S^{r} v+P, S^{r}-D v\right)
$$

and the source terms

$$
\begin{aligned}
\overrightarrow{\mathcal{S}}= & {\left[0, R_{Y_{e}}^{v},\left(S^{r} v-\tau-D\right) \alpha X\left(8 \pi r P+\frac{m}{r^{2}}\right)+\alpha P X \frac{m}{r^{2}}\right.} \\
& \left.+\frac{2 \alpha P}{X r}+Q_{S^{r}}^{\nu, \mathrm{E}}+Q_{S^{r}}^{\nu, \mathrm{M}}, Q_{\tau}^{\nu, \mathrm{E}}+Q_{\tau}^{\nu, \mathrm{M}}\right]
\end{aligned}
$$

where the $R \mathrm{~s}$ and $Q \mathrm{~s}$ are neutrino sources and sinks which arise from the neutrino leakage scheme and neutrino pressure contributions (see O'Connor \& Ott 2010 for details).

The evolution equations (Equation 5) are first spatially discritized using a finite-volume scheme (e.g., Romero et al. 1996; Font 2008). The piecewise parabolic method (Colella $\&$ Woodward 1984) is used to reconstruct the state variables at the cell interfaces and the HLLE Riemann solver (Einfeldt 1988) is employed to determine the physical fluxes through these interfaces. The evolution equations are integrated forward in time via the method of lines (Hyman 1976), using standard second-order Runge-Kutta time integration with a Courant factor of 0.5 . After updating the conserved variables, they are inverted via a Newton-Raphson scheme to obtain the new fluid state variables.

In spherical symmetry, rotation can be included by assuming constant angular velocity $\Omega$ on spherical shells (shellular rotation) and including an angle-averaged centrifugal force in the radial momentum equation. This is common practice in stellar evolution codes (e.g., Heger et al. 2000) an has also been applied to Newtonian 1D stellar collapse calculations (Akiyama et al. 2003; Thompson et al. 2005). We include a GR variant of this "1.5D" rotation treatment in GR1D (1) by adding an evolution equation for the generalized specific angular momentum $S_{\phi}=\rho h W^{2} v_{\varphi} r$, (2) by including an effective centrifugal force in the equation for $S^{r}$, and (3) by modifying the expressions for the 4-velocity, the Lorentz factor, and the differential equation for the metric potential to account for rotation. Full details as well as a demonstration of conservation of angular momentum can be found in O'Connor \& Ott (2010). Note that, as may be expected and was demonstrated by Ott et al. (2006), the 1.5D approximation becomes less accurate with increasing spin and quantitative results are reliable only for low rotation rates.

\subsection{Neutrino Treatment}

Neutrino effects are crucial in stellar collapse and should ideally be included via a computationally expensive GR Boltzmann transport treatment (e.g., Liebendörfer et al. 2004). However, since our aim is to perform an extensive parameter study with hundreds of simulations, we choose to resort to a less accurate, but much more computationally efficient leakage and approximate heating scheme for neutrinos. Details of this are laid out in O'Connor \& Ott (2010). Here we review only its most salient features.

Before core bounce, neutrinos deleptonize the collapsing core, reducing the electron fraction $Y_{e}$ and, as a consequence, the size of the homologous inner core (Bethe 1990). Liebendörfer (2005) showed that the prebounce $Y_{e}$ can be parameterized as a function of density and that this parameterization varies little between progenitor stars. We follow this prescription for prebounce deleptonization and use the $Y_{e}(\rho)$ fit parameters of his G15 model. Following bounce, this simple parameterization becomes inaccurate and cannot capture the effects of neutrino cooling, deleptonization, and neutrino heating. Hence, we switch to a leakage scheme that uses elements of what was laid out by Ruffert et al. (1996) and Rosswog \& Liebendörfer (2003). We consider three neutrino species, $v_{e}, \bar{v}_{e}$, and $v_{x}=\left\{v_{\mu}, \bar{v}_{\mu}, v_{\tau}, \bar{v}_{\tau}\right\}$. Neutrino pairs of all species are made in thermal processes of which we include electron-positron pair annihilation and plasmon decay (Ruffert et al. 1996). In addition, charged-current processes lead to the emission of $v_{e} \mathrm{~s}$ and $\bar{v}_{e} \mathrm{~s}$. The leakage scheme provides approximate energy and number emission rates that are inserted into GR1D's evolution equations via source terms in Equation (8), $R_{Y_{e}}^{v}, Q_{S^{r}}^{\nu, \mathrm{E}}$, and $Q_{\tau}^{\nu, \mathrm{E}}$ (O'Connor \& Ott 2010).

We include neutrino heating via a parameterized chargedcurrent heating scheme based on Janka (2001). The heating rate at radius $r$ is

$$
Q_{v_{i}}^{\text {heat }}(r)=f_{\text {heat }} \frac{L_{v_{i}}(r)}{4 \pi r^{2}} \sigma_{\text {heat }, v_{i}} \frac{\rho}{m_{u}} X_{i}\left\langle\frac{1}{F_{v_{i}}}\right\rangle e^{-2 \tau_{v_{i}}},
$$

where $f_{\text {heat }}$ is a scale factor that allows for artificially increased heating, $L_{v_{i}}(r)$ is the neutrino luminosity interior to $r, \tau_{v_{i}}$ is the optical depth, determined through the leakage scheme, $\sigma_{\text {heat }, v_{\mathrm{i}}}$ is the energy-averaged absorption cross section, and $X_{i}$ is corresponding mass fraction of the neutrino reaction $\left(X_{p}\right.$ for $\bar{v}_{e}$ capture on protons and $X_{n}$ for $v_{e}$ capture on neutrons). $\left\langle 1 / F_{v_{i}}\right\rangle$ is the mean inverse flux factor which we approximate analytically as a function of the optical depth $\tau$ by comparing to the angledependent radiation transport calculations of Ott et al. (2008).

We include in our simulations the stabilizing effect of neutrino pressure in the optically thick PNS core via an ideal Fermigas approximation (Liebendörfer et al. 2005; O'Connor \& Ott 2010). Leaving out this pressure contribution leads to $\sim 5 \%$ smaller maximum gravitational PNS masses. We also include terms due to neutrino pressure and radiation-field energy in the calculation of the gravitational mass (Equation (3)) and of the metric potential (Equation (4)). Since our leakage scheme does not treat neutrino energy separately from the internal energy of the fluid, including the energy of the neutrino gas in the former equations is not fully consistent with our present approach. This error was discovered and corrected after all simulations were performed. However, a set of test calculations showed that the error leads to an underestimate of the maximum gravitational PNS mass of only $\sim 2 \%$ which is well within the error of the overall leakage scheme (see also Section 4.2). 


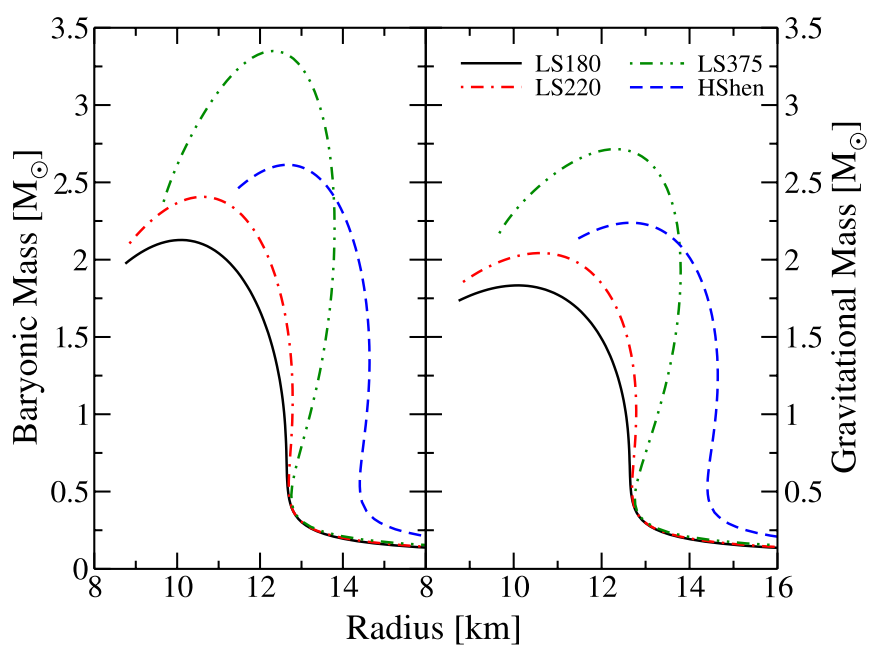

Figure 1. Baryonic (left) and gravitational (right) neutron mass-radius relations for various hot nuclear EOS. The temperature is taken to be constant throughout the star at $T=0.1 \mathrm{MeV}$ and the electron fraction is determined through neutrinoless $\beta$-equilibrium with an imposed minimum of 0.05 due to table constraints.

(A color version of this figure is available in the online journal.)

\subsection{Equations of State and Maximum Neutron Star Masses}

We include multiple finite-temperature nuclear EOS in this study to explore the dependence of postbounce evolution and $\mathrm{BH}$ formation on EOS properties. The Lattimer-Swesty (LS) EOS (Lattimer \& Swesty 1991) is based on the compressible liquid-droplet model, assumes a nuclear symmetry energy $S_{v}$ of $29.3 \mathrm{MeV}$, and comes in three variants with different values of the nuclear incompressibility of $K_{\mathrm{s}}=180 \mathrm{MeV}$ (LS180), $220 \mathrm{MeV}$ (LS220), and $375 \mathrm{MeV}$ (LS375). The EOS of Shen et al. (1998a, 1998b) (HShen EOS), on the other hand, is based on a relativistic mean-field model, has $S_{v}=36.9 \mathrm{MeV}$ and $K_{\mathrm{s}}=281 \mathrm{MeV}$. More details on these EOS and their implementation in GR1D is given in O'Connor \& Ott (2010). The EOS tables and driver routines employed in this study are available for download at http://stellarcollapse.org.

By solving the Tolman-Oppenheimer-Volkoff (TOV) equations (Oppenheimer \& Volkoff 1939) with $T=0.1 \mathrm{MeV}$ and assuming neutrinoless $\beta$-equilibrium we determine the neutron star baryonic and gravitational mass-radius relationships that each of these four EOS produces and that are depicted by Figure 1. The maximum gravitational (baryonic) neutron star masses are $\sim 1.83 M_{\odot}\left(\sim 2.13 M_{\odot}\right), \sim 2.04 M_{\odot}\left(\sim 2.41 M_{\odot}\right)$, $\sim 2.72 M_{\odot}\left(\sim 3.35 M_{\odot}\right)$, and $\sim 2.24 M_{\odot}\left(\sim 2.61 M_{\odot}\right)$ for LS180, LS220, LS375, and HShen, respectively. The coordinate radii of these maximum-mass stars are $\sim 10.1 \mathrm{~km}, \sim 10.6 \mathrm{~km}, \sim 12.3 \mathrm{~km}$ and $\sim 12.6 \mathrm{~km}$, respectively.

The above maximum neutron star masses hold only for nonrotating cold NSs. As we will discuss in detail in Section 4.3, the PNSs at the heart of the failing CCSNe considered in this work are much hotter. They have central temperatures of $\sim 10-20 \mathrm{MeV}$ and tens of $\mathrm{MeV}$ in their outer core and mantle. Thermal effects have a significant effect on their maximum masses.

In this study, we do not consider hyperonic EOS, e.g., the hyperonic extension of the HShen EOS by Ishizuka et al. (2008), or EOS involving other phases of nuclear matter, e.g., quarks and pions Nakazato et al. (2010). Such EOS are potentially interesting in failing CCSNe, since their exotic components lead to a softening of the EOS at high density, potentially accelerating
BH formation (Sumiyoshi et al. 2009). We also do not consider EOS that include QCD phase transitions that too may lead to early PNS collapse and potentially to a second bounce and neutrino burst (Sagert et al. 2009).

\section{MODEL SETUP}

\subsection{Presupernova Data}

We make use of single-star nonrotating presupernova models from several stellar evolution studies: Woosley \& Weaver (1995) (WW95), Woosley et al. (2002) (WHW02), Limongi \& Chieffi (2006) (LC06A/B), and Woosley \& Heger (2007) (WH07). Each of these studies evolved stars with a range of ZAMS masses at solar metallicity $\left(Z_{\odot}\right.$, hereafter denoted with prefix $s$ in model names) up until the onset of core collapse. In addition to solar metallicity, WHW02 evolved stars with ultra low metallicity, $10^{-4} Z_{\odot}$ (denoted by prefix $u$ ) and zero metallicity (denoted by prefix $z$ ). Rotation is of relevance in stellar evolution and stellar evolutionary processes affect the rotational configuration at the presupernova stage. In order to study $\mathrm{BH}$ formation, $\mathrm{BH}$ birth properties and their impact on a potential subsequent evolution to a GRB in such spinning progenitors, we draw representative models from Heger et al. (2000) (HLW00) and from Woosley \& Heger (2006) (WH06) who included rotation in essentially the same way as we do in GR1D.

In Table 1, we list key parameters for all models in our set. These include presupernova mass, iron core mass (which we define as the baryonic mass interior to $Y_{e}=0.495$ ), and the bounce compactness $\xi_{2.5}$. The latter is defined as

$$
\xi_{M}=\left.\frac{M / M_{\odot}}{R\left(M_{\text {bary }}=M\right) / 1000 \mathrm{~km}}\right|_{t=t_{\text {bounce }}},
$$

where we set $M=2.5 M_{\odot} . R\left(M_{\text {bary }}=2.5 M_{\odot}\right)$ is the radial coordinate that encloses $2.5 M_{\odot}$ at the time of core bounce. $\xi_{2.5}$ gives a measure of a progenitor's compactness at bounce. We choose $M=2.5 M_{\odot}$ as this is the relevant mass scale for $\mathrm{BH}$ formation. $\xi_{2.5}$ is, as we shall discuss in Section 4.4, a dimensionless variable that allows robust predictions on the postbounce dynamics and the evolution of the model toward $\mathrm{BH}$ formation. The evaluation of $\xi_{2.5}$ at core bounce is crucial, since this is the only physical and unambiguous point in core collapse at which one can define a zero of time and can describe the true initial conditions for postbounce evolution. Computing the same quantity at the precollapse stage leads to ambiguous results, since progenitors come out of stellar evolution codes in more or less collapsed states. Collapse washes out these initial conditions and removes ambiguities.

We point out (as is obvious from Table 1) that there is a clear correlation between iron core mass and bounce compactness. Since the effective Chandrasekhar mass increases due to thermal corrections (Burrows \& Lattimer 1983; Baron \& Cooperstein 1990), more massive iron cores are hotter. Hence, progenitors with greater bounce compactness result in higher-temperature PNSs.

One of the most uncertain, yet most important, variables in the evolution of massive stars is the mass-loss rate. Mass loss can vary significantly over the life of a star. Current estimates of mass loss, either theoretical or based on fits to observational data, can depend on many parameters, including mass, radius, stellar luminosity, effective surface temperature, surface hydrogen and helium abundance, and stellar metallicity (de Jager et al. 1988; Nieuwenhuijzen \& de Jager 1990; Wellstein \& Langer 1999; 
Table 1

Initial Models

\begin{tabular}{|c|c|c|c|c|}
\hline Model & $\begin{array}{c}M_{\text {ZAMS }} \\
\left(M_{\odot}\right) \\
\end{array}$ & $\begin{array}{c}M_{\text {pre-SN }} \\
\left(M_{\odot}\right) \\
\end{array}$ & $\begin{array}{c}M_{\mathrm{Fe} \text { core }}{ }^{\mathrm{a}} \\
\left(M_{\odot}\right)\end{array}$ & $\xi_{2.5^{b}}$ \\
\hline s20WW95 & 20 & 20.0 & 1.74 & 0.383 \\
\hline s25WW95 & 25 & 25.0 & 1.77 & 0.416 \\
\hline s40WW95 & 40 & 40.0 & 1.98 & 0.583 \\
\hline s15WHW02 & 15 & 12.6 & 1.55 & 0.150 \\
\hline s20WHW02 & 20 & 14.7 & 1.46 & 0.127 \\
\hline s25WHW02 & 25 & 12.5 & 1.62 & 0.326 \\
\hline s30WHW02 & 30 & 12.2 & 1.46 & 0.223 \\
\hline s35WHW02 & 35 & 10.6 & 1.49 & 0.205 \\
\hline s40WHW02 & 40 & 8.75 & 1.56 & 0.266 \\
\hline s75WHW02 & 75 & 6.36 & 1.48 & 0.112 \\
\hline u20WHW02 & 20 & 20.0 & 1.57 & 0.338 \\
\hline u25WHW02 & 25 & 25.0 & 1.53 & 0.223 \\
\hline u30WHW02 & 30 & 30.0 & 1.58 & 0.326 \\
\hline u35WHW02 & 35 & 35.0 & 1.85 & 0.664 \\
\hline u40WHW02 & 40 & 40.0 & 1.90 & 0.719 \\
\hline u45WHW02 & 45 & 44.9 & 1.96 & 0.655 \\
\hline u50WHW02 & 50 & 49.8 & 1.83 & 0.574 \\
\hline u60WHW02 & 60 & 59.6 & 1.88 & 0.623 \\
\hline u75WHW02 & 75 & 74.1 & 2.03 & 1.146 \\
\hline z20WHW02 & 20 & 20.0 & 1.48 & 0.163 \\
\hline z25WHW02 & 25 & 25.0 & 1.81 & 0.404 \\
\hline z30WHW02 & 30 & 30.0 & 1.50 & 0.221 \\
\hline z35WHW02 & 35 & 35.0 & 1.79 & 0.560 \\
\hline z40WHW02 & 40 & 40.0 & 1.90 & 0.720 \\
\hline s25LC06A & 25 & 16.2 & 1.43 & 0.204 \\
\hline s30LC06A & 30 & 12.8 & 1.48 & 0.274 \\
\hline s35LC06A & 35 & 11.8 & 1.48 & 0.242 \\
\hline s40LC06A & 40 & 12.4 & 1.50 & 0.339 \\
\hline s60LC06A & 60 & 16.9 & 1.63 & 0.603 \\
\hline s80LC06A & 80 & 22.4 & 1.67 & 0.628 \\
\hline s120LC06A & 120 & 30.5 & 1.91 & 0.905 \\
\hline s40LC06B & 40 & 6.82 & 1.51 & 0.322 \\
\hline s60LC06B & 60 & 5.95 & 1.35 & 0.163 \\
\hline s80LC06B & 80 & 6.04 & 1.46 & 0.185 \\
\hline s120LC06B & 120 & 6.12 & 1.24 & 0.143 \\
\hline s20WH07 & 20 & 15.8 & 1.55 & 0.288 \\
\hline s25WH07 & 25 & 15.8 & 1.60 & 0.334 \\
\hline s30WH07 & 30 & 13.8 & 1.49 & 0.219 \\
\hline s35WH07 & 35 & 13.6 & 1.61 & 0.369 \\
\hline s40WH07 & 40 & 15.3 & 1.83 & 0.599 \\
\hline s45WH07 & 45 & 13.0 & 1.79 & 0.556 \\
\hline s50WH07 & 50 & 9.76 & 1.50 & 0.221 \\
\hline s60WH07 & 60 & 7.25 & 1.46 & 0.175 \\
\hline s80WH07 & 80 & 6.33 & 1.48 & 0.210 \\
\hline s100WH07 & 100 & 6.04 & 1.46 & 0.247 \\
\hline s120WH07 & 120 & 5.96 & 1.43 & 0.172 \\
\hline m35OCWH06 & 35 & 28.1 & 2.08 & 0.457 \\
\hline E20HLW00 & 20 & 11.0 & 1.74 & 0.320 \\
\hline E25HLW00 & 25 & 5.45 & 1.70 & 0.294 \\
\hline
\end{tabular}

Notes. The model name contains the information necessary to uniquely specify the presupernova model. For nonrotating progenitors, the beginning letter in the model name refers to the metallicity of the progenitor, following the convention of Woosley et al. (2002), " $s$ ", " $u$ ", and " $z$ " are used for solar, $10^{-4}$ solar, and zero metallicities, respectively. Following is the ZAMS mass; next we specify the progenitor model set (see the text for references). For rotating progenitors, we follow the naming convention of the original reference.

${ }^{\text {a }}$ We define the iron core edge to be where $Y_{e}=0.495$.

b $\xi_{2.5}$ is determined at bounce in collapse runs using the LS180 EOS and will vary only slightly with EOS.

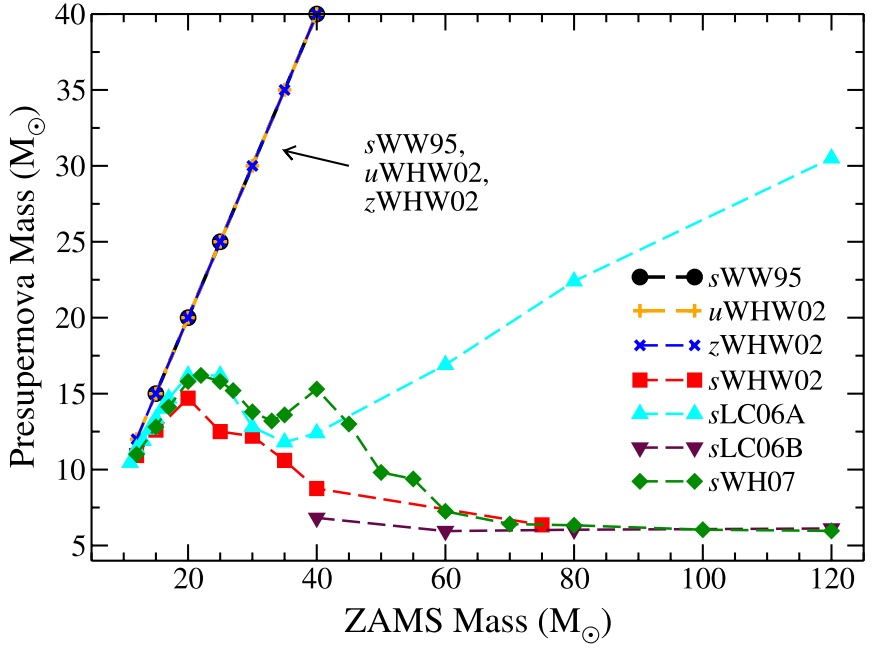

Figure 2. Presupernova mass as a function of ZAMS mass for the various model sets considered here. See the text for discussion.

(A color version of this figure is available in the online journal.)

Nugis \& Lamers 2000; Vink \& de Koter 2005). The massloss rate is uncertain in both the massive O-star and in the stripped-envelope Wolf-Rayet (W-R) star stage. O-star winds are expected to be responsible for the partial or complete removal of the hydrogen envelopes of massive stars. Recent observational results suggest that the rates used in current stellar evolution models may be too high by factors of 3-10 if clumped winds are considered correctly (Bouret et al. 2005; Fullerton et al. 2006; Puls et al. 2006). With the reduced rates, W-R stars would be difficult to make in standard single-star evolution and would require binary or eruptive mass-loss scenarios (Smith et al. 2010).

In Figure 2, we plot the mass-loss-induced mapping between ZAMS mass and presupernova mass for the ensemble of nonrotating progenitors listed in Table 1. WW95 models do not include mass loss - the presupernova models of this study have a mass equal to the ZAMS mass. WHW02 and WH07 employ the mass-loss rates of Nieuwenhuijzen \& de Jager (1990) and Wellstein \& Langer (1999) and use significantly reduced rates for low and zero metallicity stars. The $u$ and $z$ models of WHW02 have almost no mass loss and their presupernova masses are very close to their ZAMS values. The solar-metallicity stars of the $s$ WHW02 and $s$ WH07 model sets have significant mass loss, generally scaling with ZAMS mass. The most massive stars in these model sets have presupernova masses that are a small fraction of the initial ZAMS mass. For main sequence and giant phases, Limongi \& Chieffi (2006) adopt mass-loss rates following Vink et al. $(2000,2001)$ and de Jager et al. (1988). For W-R stars, they either use the massloss rates of Nugis \& Lamers (2000) (hereinafter referred to as LC06A models) or Langer (1989) (LC06B models). The latter are close to the values used for solar-metallicity stars in the WHW02 and WH07 model sets. The difference in the LC06A and LC06B mass-loss rates is roughly a factor of two. This, as portrayed by Figure 2 and evident from Table 1, can significantly alter the total mass at the onset of collapse and also has a strong effect on the iron core mass and bounce compactness.

An additional uncertainty in massive star evolution is the phenomenon of large episodic mass loss (Smith 2008). Unknowns and uncertainties in both the cause and effect of large episodic mass loss currently prevent detailed stellar evolution calculations from including this phenomenon at all. 


\subsection{Grid Setup}

Based on resolution studies, we employ a computational grid setup with a total of 1050 zones. Near the origin and extending out to $20 \mathrm{~km}$, we employ a constant grid spacing of $80 \mathrm{~m}$ (250 zones). Outside of $20 \mathrm{~km}$ we logarithmically space the remaining 800 zones to a radius where the initial density falls to $2000 \mathrm{~g} \mathrm{~cm}^{-3}$. We require the high resolution near the center for late postbounce times when the postshock region becomes small $\left(r_{\text {shock }} \lesssim 20 \mathrm{~km}\right)$ and when the PNS is close to dynamical collapse to a $\mathrm{BH}$. We interpolate the various presupernova profiles $\left(\rho, T, Y_{e}, v, \Omega\right)$ to our grid using linear interpolation.

\subsection{Rotation}

In simulations including $1.5 \mathrm{D}$ rotation, we directly use the angular velocity of the progenitor model if it was evolved with rotation or assign specific angular momentum via the rotation law

$$
j(r)=j_{16, \infty}\left[1+\left(\frac{A_{M_{\odot}}}{r}\right)^{2}\right]^{-1} 10^{16} \mathrm{~cm}^{2} \mathrm{~s}^{-1},
$$

where $j_{16, \infty}$ is the specific angular momentum at infinity in units of $10^{16} \mathrm{~cm}^{2} \mathrm{~s}^{-1}$. We define $A_{M_{\odot}}$ to be the radius where the enclosed mass is $1 M_{\odot}$. This is a variation on the rotation law commonly used in simulations of rotating core collapse (e.g., Ott et al. 2006), where $\Omega(r)=j(r) r^{-2}$ is prescribed and the differential-rotation parameter $A$ is set to some constant radius. The advantage of prescribing $j$ (which is conserved along Lagrangian trajectories) and choosing the value of $A$ based on a mass coordinate is that progenitors from different groups that are evolved to different points still yield similar PNS angular momentum distributions for a given choice of $j_{16, \infty}$. Equation (11) leads to roughly uniform rotation in the core inside $A_{M_{\odot}}\left(j(r) \propto r^{2}\right)$ and angular velocity $\Omega(r)$ decreasing with $r^{2}$ further out $\left(j(r)=\right.$ const). We note that when $1 M_{\odot}$ of material is contained within $10^{3} \mathrm{~km}$, which is typical of many progenitors, the central rotation rate is $j_{16, \infty} \mathrm{rad} \mathrm{s}^{-1}$.

Our way of assigning rotation to precollapse models approximates well the predictions of core rotation (inner $\sim$ few $M_{\odot}$ ) from stellar evolution studies (see, e.g., Ott et al. 2006 for comparison plots) and, thus, is useful for studying rotational effects on BH formation. Equation (11) does not, however, capture the rise in specific angular momentum observed at larger radii (or mass coordinate) that is important for the potential evolution toward a long GRB and seen in recent rotating progenitor models (e.g., Woosley \& Heger 2006).

\section{RESULTS}

\subsection{Fiducial Model}

We begin our discussion with a detailed description of the evolution of a failing CCSN from core collapse, through bounce, and the subsequent postbounce evolution to $\mathrm{BH}$ formation. For this, we choose the $40 M_{\odot}$ ZAMS-mass progenitor model $s 40$ WH07. We evolve this progenitor using the LS180 EOS, do not include rotation, and use the standard setting of $f_{\text {heat }}=1$ (see Section 2.2). In Figure 3, we show the evolution of the radial coordinate of select baryonic mass shells as a function of time and we highlight shells enclosing $0.5,1.0,1.5,2.0$, and $2.5 M_{\odot}$. In addition, the figure shows the shock radius and the positions of the energy-averaged $v_{e}$ and $v_{x}$ neutrinospheres as

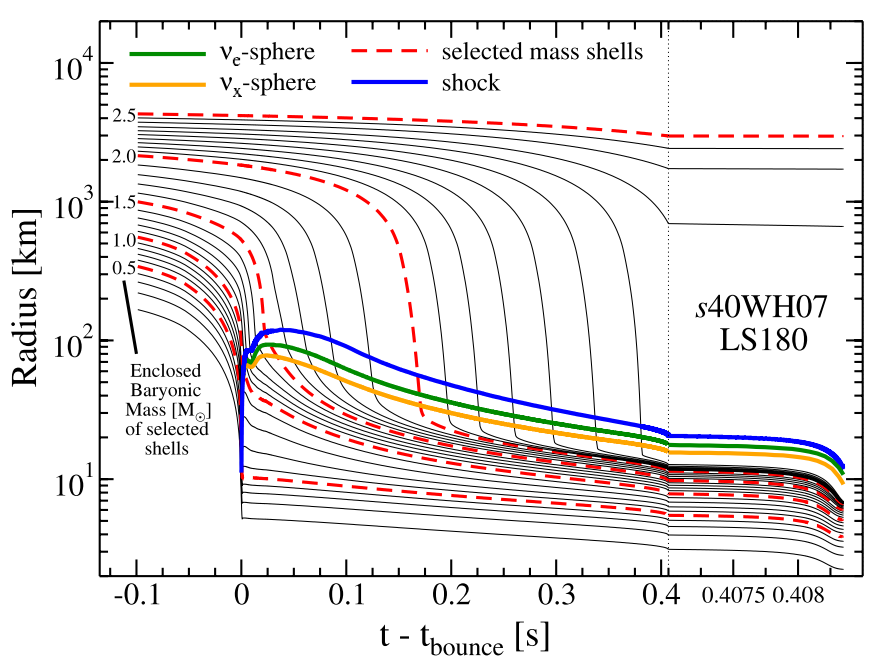

Figure 3. Evolution of baryonic mass shells in the nonrotating model $s 40 \mathrm{WH} 07$ evolved with the LS180 EOS. We also include the shock location and the radii of the $v_{e}$ and $v_{x}$ neutrinospheres. The $\bar{v}_{e}$-sphere (not shown) is inside, but very close to the $v_{e}$-sphere. The vertical dotted line denotes a change of timescale in the plot, highlighting the final $\sim 1 \mathrm{~ms}$ of evolution before the central density reaches $\sim 4.2 \times 10^{15} \mathrm{~g} \mathrm{~cm}^{-3}$ and the simulation halts. We specifically highlight the $0.5,1.0,1.5,2.0$, and $2.5 M_{\odot}$ baryonic mass shells with dashed lines. With solid lines, for $M<2 M_{\odot}$, we plot every $0.1 M_{\odot}$ mass shell. Above $2 M_{\odot}$, we plot mass shells with a spacing of $0.05 M_{\odot}$.

(A color version of this figure is available in the online journal.)

a function of time. The prebounce collapse phase $(t<0)$ lasts $\sim 450 \mathrm{~ms}$. At bounce, the central value of the lapse function is $\alpha_{c} \sim 0.82$, and the metric function $X$ has a maximum of $\sim 1.1$ and peaks off-center at a baryonic mass coordinate of $\sim 0.6 M_{\odot}$ which roughly corresponds to the edge of the inner core. The inner core initially overshoots to a maximum central density $\rho_{c} \sim 5.0 \times 10^{14} \mathrm{~g} \mathrm{~cm}^{-3}$, then settles at $\sim 3.7 \times 10^{14} \mathrm{~g} \mathrm{~cm}^{-3} \cdot \rho_{c}$ subsequently increases as accretion adds mass to the PNS. The bounce shock forms at a baryonic mass coordinate of $\sim 0.6 M_{\odot}$. From there, it moves out quickly in mass, reaching a baryonic mass coordinate of $\sim 1.5 M_{\odot}$ at $22 \mathrm{~ms}$ after bounce, $2 M_{\odot}$ at $\sim 162 \mathrm{~ms}$, and $2.25 M_{\odot}$ at $\sim 329 \mathrm{~ms}$. In radius, the shock reaches a maximum of $\sim 120 \mathrm{~km}$ at $38 \mathrm{~ms}$ after bounce. There it stalls, then slowly recedes. At $10 \mathrm{~ms}$ after bounce, the accretion rate through the shock is $\sim 18 M_{\odot} \mathrm{s}^{-1}$ and drops to $\sim 2.7, \sim 1.7$, and $\sim 1.25 M_{\odot} \mathrm{s}^{-1}$ at 100,200 , and $300 \mathrm{~ms}$ after bounce, respectively. The drop in the accretion rate has little effect on the failing supernova engine. In agreement with previous work that employed a more accurate neutrino treatment (e.g., Thompson et al. 2003; Liebendörfer et al. 2005), the 1D neutrino mechanism is manifestly ineffective in driving the shock, yielding, in this model, a heating efficiency $\eta=L_{\text {absorbed }} / L_{v_{e}+\bar{v}_{e}}$ of only $\sim 3 \%$ (on average). The neutrinospheres (where the energy-averaged optical depth $\tau=2 / 3$ ) are initially exterior to the shock but are surpassed by the latter in a matter of milliseconds after bounce, leading to the $v_{e}$ deleptonization burst. At all times, the $v_{x}$ neutrinosphere is interior to the $\bar{v}_{e}$ neutrinosphere, which in turn is slightly interior to the $v_{e}$ neutrinosphere. The mean neutrino energies also follow this order. They are the largest for $v_{x}$ and the lowest for $v_{e}$ and increase with decreasing neutrinosphere radii (e.g., Thompson et al. 2003; Sumiyoshi et al. 2007; Ott et al. 2008; Fischer et al. 2009).

At $\sim 408 \mathrm{~ms}$ after bounce, the shock has receded to $\sim 20 \mathrm{~km}$ and the PNS has reached a baryonic (gravitational) mass of $\sim 2.33 M_{\odot}\left(\sim 2.23 M_{\odot}\right)$. The difference between baryonic and 
gravitational mass, at this point in the evolution, is due to the $\sim 1.9 \times 10^{53}$ erg of energy radiated by neutrinos. At this point, dynamical PNS collapse to a $\mathrm{BH}$ sets in and happens on a coordinate timescale of $\lesssim 1 \mathrm{~ms}$. In the rightmost part of Figure 3, we zoom in to the final $1 \mathrm{~ms}$ of evolution to show detail. The first signs of collapse manifest themselves in the development of a radial infall velocity profile at the PNS edge. The PNS then collapses in on itself and the central density increases by a factor of $\sim 3$ in only $\sim 1 \mathrm{~ms}$ of coordinate time. The simulation crashes due to EOS limitations at $\rho_{c} \sim$ $4.2 \times 10^{15} \mathrm{~g} \mathrm{~cm}^{-3}$ and with $\alpha_{c}=0.006$. At this point the peak of the metric function $X=[1-2 m(r) / r]^{-1 / 2}$ is $\sim 4.4$ at a coordinate radius of $\sim 6.8 \mathrm{~km}$. There, the fluid velocity also peaks at $\sim-0.83 c$. The shock recedes by $\sim 8 \mathrm{~km}$ in the last $\sim 1 \mathrm{~ms}$ of evolution to a radial coordinate of $\sim 12 \mathrm{~km}$. During the last $\sim 0.05 \mathrm{~ms}$, due to the central lapse dropping to nearly zero, the evolution of the mass shells slows near the origin. This is characteristic for our choice of gauge. If the simulation were to continue, $X$ would become singular at the event horizon that would appear after infinite coordinate time in our coordinates (Petrich et al. 1986).

The $s 40 \mathrm{WH} 07$ model discussed here is a typical example of a failing CCSN in spherical symmetry. We present the results of a large number of such models in Table 2, where for each EOS and progenitor model we show the time to $\mathrm{BH}$ formation as measured from bounce and the mass, both baryonic and gravitational, of the PNS when the central value of the lapse function $\alpha$ reaches 0.3 (roughly the point of instability). In this table, the model name describes the initial model. The metallicity is denoted by one of three letters: $s, u$, and $z$ which represent solar, $10^{-4}$ solar, and zero metallicity, respectively. Following the metallicity is the ZAMS mass and the progenitor model set. In many simulations, particularly in those employing stiff EOS, a BH does not form within $3.5 \mathrm{~s}$. For these simulations we include in parentheses the mass inside the shock at $3.5 \mathrm{~s}$. We note that at $\mathrm{BH}$ formation the shock is typically at a distance of $\lesssim 20 \mathrm{~km}$ and there is very little mass between the shock and the PNS. The dynamical collapse to a BH happens very quickly $(t \lesssim 1 \mathrm{~ms})$ during which very little additional accretion occurs.

\subsection{Comparison with Previous Work}

The $s 40 \mathrm{WW} 95$ progenitor was considered in the $\mathrm{BH}$ formation studies of Liebendörfer et al. (2004), Sumiyoshi et al. (2007) (hereinafter referred to as S07), and Fischer et al. (2009) (hereinafter referred to as F09). For comparison, we perform simulations with this progenitor for both the LS180 and HShen EOS. Table 3 compares two key quantities, the time to $\mathrm{BH}$ formation and the maximum baryonic PNS mass, obtained with GR1D with the results obtained in the aforementioned studies.

For the LS180 EOS, we find a time to BH formation of $\sim 524 \mathrm{~ms}$ and a maximum baryonic PNS mass of $\sim 2.26 \mathrm{M}_{\odot}$, which is $\sim 3 \%$ larger than predicted by F09. We attribute this discrepancy to the different neutrino transport methods used. GR1D's leakage scheme has the tendency to somewhat overpredict electron-type neutrino luminosities (see the discussion in O'Connor \& Ott 2010), resulting in lower gravitational masses compared to full Boltzmann transport calculations. Our time to $\mathrm{BH}$ formation is longer by $\sim 100 \mathrm{~ms}$ or $\sim 20 \%$. This disagreement is relatively larger than the baryonic mass disagreement due to the low accretion rate at late times that translates small differences in mass to large differences in time. At $\sim 435.5 \mathrm{~ms}$, the time to $\mathrm{BH}$ formation of F09, our PNS has a baryonic mass of $\sim 2.17 M_{\odot}$, which is consistent to $\sim 1 \%$ with F09. We find it more difficult to reconcile our results (and those of Liebendörfer et al. (2004) and F09) with the simulations of S07. Their maximum PNS baryonic mass and the time to $\mathrm{BH}$ formation suggest a lower accretion rate throughout their evolution $\left(\sim 2.1 M_{\odot}\right.$ in $\sim 560 \mathrm{~ms}$ ).

In the simulation run with the stiffer HShen EOS, the larger maximum PNS mass leads to a delay of $\mathrm{BH}$ formation until a postbounce time $\sim 1.129$ s and we find a maximum baryonic PNS mass of $\sim 2.82 M_{\odot}$. The maximum PNS mass and time to $\mathrm{BH}$ formation of S07 again suggest an accretion rate in disagreement with F09 and our work. The results of F09 with the HShen EOS suffer from a glitch in F09's EOS table interpolation scheme which has since been fixed (T. Fischer 2010, private communication). This leads to a postbounce time to $\mathrm{BH}$ formation of $\sim 1.4 \mathrm{~s}$ and a maximum baryonic PNS mass of $\sim 3.2 M_{\odot}$. Results from more recent simulations correct this error and are presented in Table 3 (T. Fischer 2010, private communication).

\subsection{Equation-of-state Dependence and Thermal Effects}

The maximum PNS mass and, thus, the evolution toward $\mathrm{BH}$ formation, depends strongly on the EOS. This was realized early on (Burrows 1988) and has recently been investigated by S07 and F09 who compared models evolved with the LS180 and HShen EOS. Here we extend their discussion and include also the LS220 and LS375 EOS. For a given accretion history, set by progenitor structure and independent of the high-density EOS, a stiffer nuclear EOS leads to a larger postbounce time to $\mathrm{BH}$ formation. In Figure 4, we plot the evolution of the central density $\rho_{c}$ of the $s 40 \mathrm{WH} 07$ model evolved with the four considered EOS. Each EOS leads to a characteristic maximum central density at bounce that is practically independent of progenitor model: $\sim 4.8 \times 10^{14} \mathrm{~g} \mathrm{~cm}^{-3}, \sim 4.4 \times 10^{14} \mathrm{~g} \mathrm{~cm}^{-3}$, $\sim 3.7 \times 10^{14} \mathrm{~g} \mathrm{~cm}^{-3}$, and $\sim 3.4 \times 10^{14} \mathrm{~g} \mathrm{~cm}^{-3}$ for the LS180, LS220, LS375, and HShen EOS, respectively. As expected, the variant using the softest nuclear EOS (LS180) shows the steepest postbounce increase in $\rho_{c}$ and becomes unstable to $\mathrm{BH}$ formation at only $\sim 408 \mathrm{~ms}$ for this progenitor. The onset of $\mathrm{BH}$ formation is marked by a quick rise in the central density. This is most obvious from the $\rho_{c}$ evolutions of the model variants using the stiff HShen and LS375 EOS.

Interestingly, the nominally stiffest EOS (LS375) leads to higher central densities than the softer HShen EOS up until $\sim 1.1 \mathrm{~s}$ after bounce. We find that this is due to the HShen EOS yielding higher pressure at $\rho \lesssim 3 \times 10^{14} \mathrm{~g} \mathrm{~cm}^{-3}, T \sim 10 \mathrm{MeV}$, and $Y_{e} \sim 0.3$. This higher pressure, initially in the core and later in the outer PNS layers, maintains the PNS at a lower central density. The cold-NS mass-radius relation shown in Figure 1 also exhibits this. For a given low-mass NS, the HShen EOS predicts a lower central density. For cold NSs, this trend continues until $\rho_{c} \sim 5.4 \times 10^{14} \mathrm{~g} \mathrm{~cm}^{-3}$. Thermal effects, which are stronger in the HShen EOS, will increase this value for hot PNSs.

We also plot in Figure 4 the evolution of the mass accretion rate $\dot{M}$ in model $s 40 \mathrm{WH} 07$ (evaluated at a radius of $200 \mathrm{~km}$ ). Variations in the high-density EOS have no effect on $\dot{M}$ which is most sensitive to progenitor structure. Sudden drops in $\dot{M}$ occur when density discontinuities that go along with compositional interfaces advect in. An example of this can be seen in $s 40 \mathrm{WH} 07$ at $\sim 400 \mathrm{~ms}$ after bounce where $\dot{M}$ drops by $\sim 30 \%$ due to a density jump at a baryonic mass coordinate of $\sim 2.35 M_{\odot}$. Such interfaces are common features of evolved massive stars (Woosley et al. 2002) and can help jumpstart shock revival in 
Table 2

Black Hole Formation Properties

\begin{tabular}{|c|c|c|c|c|c|c|c|c|c|c|c|c|}
\hline \multirow[t]{2}{*}{ Model } & \multicolumn{3}{|c|}{ LS180 } & \multicolumn{3}{|c|}{ LS220 } & \multicolumn{3}{|c|}{ LS375 } & \multicolumn{3}{|c|}{ HShen } \\
\hline & $\begin{array}{l}t_{\mathrm{BH}} \\
(\mathrm{s})\end{array}$ & $\begin{array}{c}M_{\mathrm{b}, \max } \\
\left(M_{\odot}\right)\end{array}$ & $\begin{array}{c}M_{\mathrm{g}, \max } \\
\left(M_{\odot}\right)\end{array}$ & $\begin{array}{l}t_{\mathrm{BH}} \\
(\mathrm{s})\end{array}$ & $\begin{array}{c}M_{\mathrm{b}, \max } \\
\left(M_{\odot}\right)\end{array}$ & $\begin{array}{c}M_{\mathrm{g}, \max } \\
\left(M_{\odot}\right)\end{array}$ & $\begin{array}{l}t_{\mathrm{BH}} \\
(\mathrm{s})\end{array}$ & $\begin{array}{c}M_{\mathrm{b}, \max } \\
\left(M_{\odot}\right)\end{array}$ & $\begin{array}{c}M_{\mathrm{g}, \max } \\
\left(M_{\odot}\right)\end{array}$ & $\begin{array}{l}t_{\mathrm{BH}} \\
(\mathrm{s})\end{array}$ & $\begin{array}{c}M_{\mathrm{b}, \max } \\
\left(M_{\odot}\right)\end{array}$ & $\begin{array}{c}M_{\mathrm{g}, \max } \\
\left(M_{\odot}\right)\end{array}$ \\
\hline s20WW95 & 0.787 & 2.238 & 2.108 & 1.129 & 2.377 & 2.201 & 3.351 & 3.060 & 2.653 & 2.287 & 2.751 & 2.486 \\
\hline s25WW95 & 0.737 & 2.246 & 2.118 & 1.046 & 2.383 & 2.211 & 2.707 & 3.054 & 2.656 & 1.990 & 2.760 & 2.498 \\
\hline s40WW95 & 0.524 & 2.263 & 2.137 & 0.666 & 2.406 & 2.240 & 1.381 & 3.043 & 2.674 & 1.129 & 2.815 & 2.562 \\
\hline s20WHW02 & & (1.949) & (1.794) & $\ldots$ & (1.950) & (1.798) & $\ldots$ & (1.951) & (1.807) & & (1.943) & $(1.805)$ \\
\hline s25WHW02 & 1.021 & 2.211 & 2.079 & 1.504 & 2.355 & 2.172 & $\ldots$ & (2.917) & (2.559) & 2.929 & 2.736 & 2.468 \\
\hline s30WHW02 & 1.820 & 2.144 & 1.978 & 2.986 & 2.331 & 2.108 & $\ldots$ & (2.416) & (2.182) & $\ldots$ & $(2.405)$ & $(2.190)$ \\
\hline s35WHW02 & 2.073 & 2.141 & 1.976 & 3.334 & 2.328 & 2.105 & $\ldots$ & $(2.351)$ & (2.137) & $\ldots$ & $(2.340)$ & (2.141) \\
\hline s40WHW02 & 1.512 & 2.168 & 2.019 & 2.231 & 2.336 & 2.134 & $\ldots$ & (2.634) & (2.355) & $\ldots$ & $(2.615)$ & $(2.362)$ \\
\hline s75WHW02 & $\ldots$ & (1.920) & $(1.781)$ & $\ldots$ & (1.920) & (1.784) & $\ldots$ & (1.921) & (1.791) & $\ldots$ & (1.913) & (1.787) \\
\hline u20WHW02 & 0.938 & 2.215 & 2.082 & 1.367 & 2.358 & 2.175 & $\ldots$ & $(2.852)$ & (2.516) & 3.004 & 2.734 & 2.466 \\
\hline u25WHW02 & 1.759 & 2.160 & 2.009 & 2.798 & 2.330 & 2.124 & $\ldots$ & (2.446) & (2.218) & & $(2.429)$ & $(2.220)$ \\
\hline u30WHW02 & 0.922 & 2.217 & 2.084 & 1.353 & 2.359 & 2.178 & $\ldots$ & $(2.802)$ & (2.483) & 3.228 & 2.731 & 2.462 \\
\hline u35WHW02 & 0.379 & 2.347 & 2.242 & 0.484 & 2.465 & 2.329 & 1.308 & 3.020 & 2.693 & 1.075 & 2.847 & 2.608 \\
\hline u40WHW02 & 0.369 & 2.346 & 2.241 & 0.453 & 2.469 & 2.333 & 0.946 & 3.023 & 2.710 & 0.849 & 2.874 & 2.638 \\
\hline u45WHW02 & 0.441 & 2.301 & 2.187 & 0.548 & 2.433 & 2.284 & 1.108 & 3.027 & 2.694 & 0.959 & 2.842 & 2.600 \\
\hline u50WHW02 & 0.563 & 2.273 & 2.154 & 0.706 & 2.408 & 2.251 & 1.365 & 3.030 & 2.676 & 1.163 & 2.816 & 2.569 \\
\hline u60WHW02 & 0.432 & 2.363 & 2.267 & 0.579 & 2.460 & 2.331 & 1.346 & 3.009 & 2.693 & 1.165 & 2.849 & 2.620 \\
\hline u75WHW02 & 0.226 & 2.526 & 2.449 & 0.285 & 2.592 & 2.498 & 0.626 & 3.006 & 2.775 & 0.594 & 2.984 & 2.791 \\
\hline z20WHW02 & 3.295 & 2.116 & 1.934 & 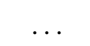 & $(2.141)$ & (1.955) & $\ldots$ & $(2.143)$ & $(1.968)$ & & $(2.132)$ & $(1.968)$ \\
\hline z25WHW02 & 0.602 & 2.283 & 2.167 & 0.956 & 2.398 & 2.239 & 3.443 & 3.050 & 2.650 & 2.351 & 2.762 & 2.505 \\
\hline z30WHW02 & 1.772 & 2.149 & 1.989 & 2.964 & 2.329 & 2.114 & $\ldots$ & (2.413) & (2.187) & $\ldots$ & $(2.401)$ & (2.192) \\
\hline z35WHW02 & 0.446 & 2.321 & 2.213 & 0.619 & 2.436 & 2.291 & 1.939 & 3.027 & 2.669 & 1.380 & 2.813 & 2.569 \\
\hline z40WHW02 & 0.365 & 2.350 & 2.245 & 0.450 & 2.471 & 2.335 & 0.958 & 3.023 & 2.711 & 0.856 & 2.874 & 2.639 \\
\hline s25LC06A & 1.220 & 2.176 & 2.029 & 2.547 & 2.333 & 2.130 & $\ldots$ & $(2.440)$ & $(2.213)$ & 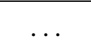 & $(2.398)$ & $(2.195)$ \\
\hline s30LC06A & 1.101 & 2.181 & 2.035 & 1.726 & 2.342 & 2.141 & $\ldots$ & (2.767) & (2.446) & & (2.695) & $(2.421)$ \\
\hline s35LC06A & 1.029 & 2.186 & 2.040 & 1.726 & 2.338 & 2.133 & $\ldots$ & (2.567) & (2.305) & & $(2.517)$ & $(2.285)$ \\
\hline s40LC06A & 0.746 & 2.232 & 2.102 & 1.138 & 2.372 & 2.193 & $\ldots$ & (2.796) & $(2.470)$ & 3.390 & 2.723 & 2.452 \\
\hline s60LC06A & 0.393 & 2.331 & 2.224 & 0.512 & 2.450 & 2.310 & 1.536 & 3.025 & 2.678 & 1.278 & 2.816 & 2.572 \\
\hline s80LC06A & 0.429 & 2.308 & 2.197 & 0.530 & 2.437 & 2.293 & 1.075 & 3.021 & 2.689 & 1.083 & 2.825 & 2.581 \\
\hline s120LC06A & 0.262 & 2.439 & 2.351 & 0.317 & 2.531 & 2.423 & 0.661 & 3.001 & 2.745 & 0.728 & 2.911 & 2.701 \\
\hline s40LC06B & 0.958 & 2.189 & 2.043 & 1.411 & 2.349 & 2.152 & $\ldots$ & $(2.957)$ & $(2.576)$ & 2.887 & 2.720 & 2.444 \\
\hline s60LC06B & 3.073 & 2.117 & 1.934 & $\ldots$ & (2.166) & $(1.972)$ & $\ldots$ & $(2.165)$ & (1.984) & $\ldots$ & $(2.126)$ & $(1.961)$ \\
\hline s80LC06B & 2.441 & 2.131 & 1.963 & $\ldots$ & $(2.260)$ & (2.052) & $\ldots$ & (2.264) & (2.071) & & $(2.249)$ & (2.069) \\
\hline s120LC06B & 2.983 & 2.120 & 1.944 & $\ldots$ & $(2.171)$ & (1.984) & $\ldots$ & (2.167) & (1.992) & $\ldots$ & (2.102) & (1.947) \\
\hline s20WH07 & 1.275 & 2.180 & 2.035 & 1.876 & 2.341 & 2.143 & $\ldots$ & $(2.712)$ & $(2.412)$ & & $(2.694)$ & $(2.426)$ \\
\hline s25WH07 & 1.066 & 2.202 & 2.065 & 1.523 & 2.352 & 2.165 & $\ldots$ & (2.975) & (2.595) & 2.796 & 2.736 & 2.466 \\
\hline s30WH07 & 1.751 & 2.150 & 1.991 & 2.978 & 2.329 & 2.115 & $\ldots$ & (2.408) & (2.184) & $\ldots$ & (2.397) & $(2.190)$ \\
\hline s35WH07 & 0.836 & 2.232 & 2.104 & 1.203 & 2.369 & 2.194 & & (2.918) & $(2.563)$ & 2.689 & 2.744 & 2.481 \\
\hline s40WH07 & 0.408 & 2.334 & 2.228 & 0.561 & 2.448 & 2.306 & 1.596 & 3.024 & 2.680 & 1.259 & 2.827 & 2.585 \\
\hline s45WH07 & 0.454 & 2.319 & 2.210 & 0.626 & 2.435 & 2.289 & 2.027 & 3.028 & 2.667 & 1.395 & 2.812 & 2.567 \\
\hline s50WH07 & 1.813 & 2.147 & 1.987 & 2.989 & 2.329 & 2.113 & $\ldots$ & $(2.411)$ & $(2.185)$ & $\ldots$ & (2.399) & $(2.190)$ \\
\hline s60WH07 & 2.778 & 2.124 & 1.947 & $\ldots$ & $(2.230)$ & (2.023) & $\ldots$ & $(2.232)$ & (2.039) & $\ldots$ & $(2.220)$ & $(2.040)$ \\
\hline s80WH07 & 2.113 & 2.139 & 1.974 & 3.284 & 2.328 & 2.104 & & $(2.363)$ & $(2.145)$ & & $(2.350)$ & (2.148) \\
\hline s100WH07 & 1.457 & 2.163 & 2.008 & 2.355 & 2.335 & 2.124 & $\ldots$ & (2.539) & $(2.281)$ & & $(2.524)$ & (2.289) \\
\hline s120WH07 & 3.043 & 2.120 & 1.940 & $\ldots$ & (2.179) & (1.985) & $\ldots$ & (2.180) & (1.999) & $\ldots$ & (2.169) & (1.999) \\
\hline
\end{tabular}

Notes. BH formation times and maximum PNS mass (both baryonic and gravitational) for nonrotating runs with $f_{\text {heat }}=1$ for all four EOS. We stop our simulations at $3.5 \mathrm{~s}$ after core bounce. Models that have not formed a BH by then probably explode in nature. They are marked by $\cdots$, but we include the masses inside the shock at $3.5 \mathrm{~s}$ in parentheses. The progenitor models are the result of various stellar evolution studies: WW95, Woosley \& Weaver (1995); WHW02, Woosley et al. (2002); LC06, Limongi \& Chieffi (2006); and WH07, Woosley \& Heger (2007).

special cases (see, e.g., the 11.2 $M_{\odot}$ model of Buras et al. 2006a, and Section 4.5 of this study). In the BH-formation context, they lead to a disproportionate increase in the time to $\mathrm{BH}$ formation in models whose EOS permit a PNS with mass greater than the mass coordinate of the density jump.

The maximum gravitational (baryonic) PNS masses for the four models shown in Figure 4 are $\sim 2.23 M_{\odot}$ $\left(\sim 2.33 M_{\odot}\right), \sim 2.31 M_{\odot}\left(\sim 2.45 M_{\odot}\right), \sim 2.68 M_{\odot}\left(\sim 3.02 M_{\odot}\right)$, and $\sim 2.59 M_{\odot}\left(\sim 2.83 M_{\odot}\right)$; and the BH formation times are $\sim 408 \mathrm{~ms}, \sim 561 \mathrm{~ms}, \sim 1.596 \mathrm{~s}$, and $\sim 1.259 \mathrm{~s}$ for the LS180, LS220, LS375, and HShen EOS, respectively. The maximum cold NS gravitational masses are $\sim 1.83 M_{\odot}, \sim 2.04 M_{\odot}$, $\sim 2.72 M_{\odot}$, and $\sim 2.24 M_{\odot}$ for the LS180, LS220, LS375, and HShen EOS, respectively.

In models evolved with the LS180, LS220, and HShen EOS, the maximum gravitational PNS mass is larger than the 


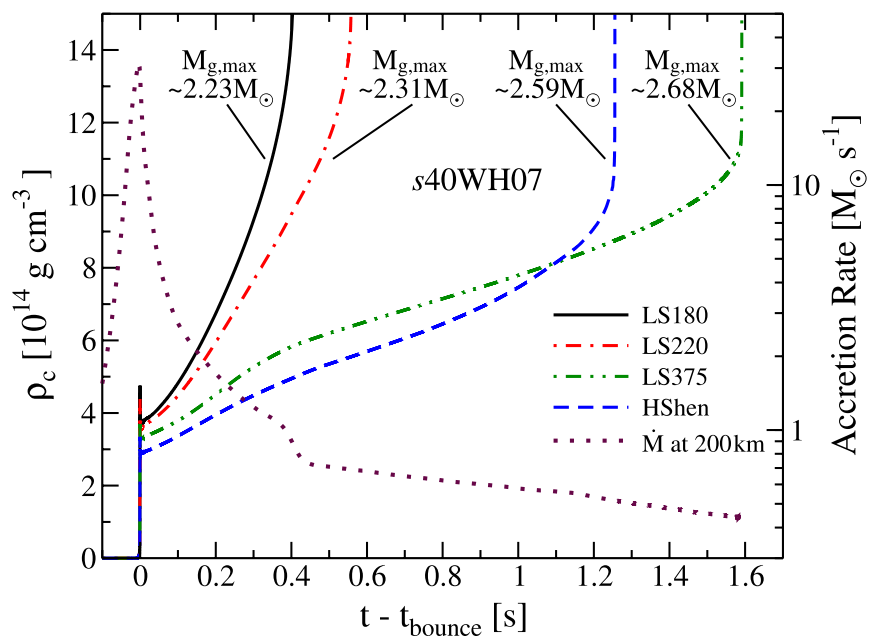

Figure 4. Central density (left ordinate) and accretion rate (right ordinate) vs. time since bounce for the $s 40 \mathrm{WH} 07$ progenitor and four EOS. BH formation occurs when the central density diverges. Each $\rho_{c}$ curve is annotated with the maximum gravitational PNS mass. The drop in the accretion rate at $t \sim 0.4 \mathrm{~s}$ is due to the accretion of a mass shell where the density drops by $\sim 30 \%$. Note the accretion rate is on a logarithmic scale.

(A color version of this figure is available in the online journal.)

Table 3

s40WW95 Comparison with LS180 and HShen EOS

\begin{tabular}{|c|c|c|c|c|}
\hline \multirow[t]{2}{*}{ Study } & \multicolumn{2}{|c|}{ LS180 } & \multicolumn{2}{|c|}{ HShen } \\
\hline & $\begin{array}{c}t_{\mathrm{BH}} \\
(s)\end{array}$ & $\begin{array}{c}M_{b, \max } \\
\left(M_{\odot}\right)\end{array}$ & $\begin{array}{c}t_{\mathrm{BH}} \\
(s)\end{array}$ & $\begin{array}{c}M_{b, \max } \\
\left(M_{\odot}\right)\end{array}$ \\
\hline Liebendörfer et al. (2004) & $\sim 0.5$ & $\sim 2.20$ & $\ldots$ & $\ldots$ \\
\hline Sumiyoshi et al. (2007) & 0.56 & 2.1 & 1.34 & 2.66 \\
\hline Fischer et al. (2009) & 0.4355 & 2.196 & $1.030^{\mathrm{a}}$ & $2.866^{\mathrm{a}}$ \\
\hline This work & 0.524 & 2.263 & 1.129 & 2.815 \\
\hline
\end{tabular}

Note. ${ }^{a}$ See the text for a discussion of the HShen EOS results from Fischer et al. (2009).

maximum gravitational cold NS mass. We can understand the differences between these cold NS and PNS maximum masses by comparing the PNS structure with various TOV solutions. In Figure 5, we plot the density and temperature profiles of the $s$ 40WH07 model evolved with the HShen EOS just prior to collapse to a BH. At this time, $t \sim 1.098 \mathrm{~s}$, the central lapse is $\alpha_{c}=0.35$, the central density is $\rho_{c} \sim 1.44 \times 10^{15} \mathrm{~g} \mathrm{~cm}^{-3}$, $T_{c} \sim 42.4 \mathrm{MeV}$, and the PNS gravitational (baryonic) mass is $\sim 2.51 M_{\odot}\left(\sim 2.74 M_{\odot}\right)$. For comparison, we include in Figure 5 three TOV solutions, all with the same central density but different temperature and $Y_{e}$ profiles. Specifically, we plot the density profile assuming (1) $T(r)=0.1 \mathrm{MeV}$, this is the "cold" NS case, (2) $T(r)=42.4 \mathrm{MeV}$, which corresponds to the central temperature from the GR1D evolution, and (3) $T(r)=T_{\mathrm{GR} 1 \mathrm{D}}$, assuming the same radial temperature profile as the GR1D model. We impose neutrinoless $\beta$-equilibrium for the former two TOV solutions and, similar to the temperature, assume the $Y_{e}$ profile of the GR1D model for the latter. For this comparison, GR1D is run without neutrino pressure and energy contributions, since they are also neglected in the TOV solution.

Inside of $\sim 6 \mathrm{~km}$, corresponding to a gravitational mass coordinate of $\sim 0.4 M_{\odot}$, the material is not shock heated but rather is heated only via adiabatic compression. The outer regions $(\sim 6-11 \mathrm{~km})$ of the PNS are hot compared to the inner core. This is due to accretion and compression of shock heated material onto the PNS surface. In this region, the thermal

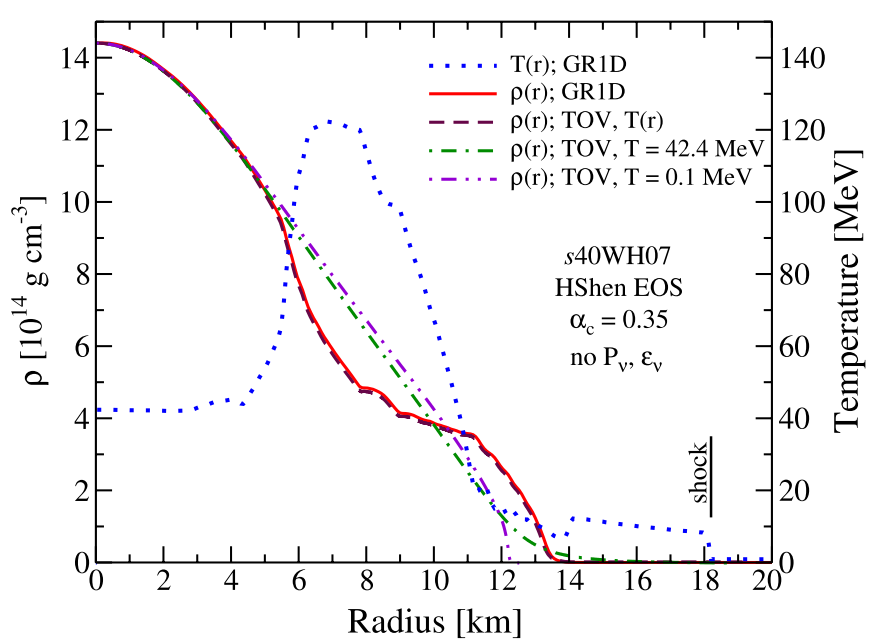

Figure 5. Comparison of radial density (left ordinate) and temperature (right ordinate) profiles of the PNS just before collapse $\left(\alpha_{c}=0.35\right)$ in model s40WH07 evolved with the HShen EOS with profiles obtained from a TOV solution using the same central density and the same radial temperature and $Y_{e}$ distributions as in model $s 40 \mathrm{WH} 07$ (dashed). For comparison, we also include profiles obtained with the TOV equations assuming both $T=T_{c}=42.4 \mathrm{MeV}$ (dot-dashed) and $T=0.1 \mathrm{MeV}$ "cold" (dot-dot-dashed) and $\beta$-equilibrium. The flattening of the density profile between 5 and $11 \mathrm{~km}$ is due to the strong thermal pressure support in this region (dotted). The gravitational mass inside the shock (whose position we denote with a vertical black line) of the $s 40 \mathrm{WH} 07$ model and of the TOV star agree to within $2 \%$. For this comparison, we switched off neutrino contributions to the internal energy and pressure in GR1D.

(A color version of this figure is available in the online journal.)

pressure support is sufficiently strong to flatten out the PNS density profile. More mass is located at larger radii compared to constant-temperature TOV solutions. This decreases PNS compactness, increasing the maximum gravitational mass. The cold-NS and the $T=T_{c}$ TOV solutions have a gravitational mass of $\sim 2.23 M_{\odot}$ and $\sim 2.35 M_{\odot}$, respectively. On the other hand, the TOV solution that assumes the same $T$ and $Y_{e}$ profile as the GR1D model yields a gravitational mass of $\sim 2.46 M_{\odot}$, within $\sim 2 \%$ of the PNS gravitational mass in the full GR1D simulation. Tests in which we vary the $Y_{e}$ distribution in the TOV solution with $T=T(r)$ show that the maximum PNS mass is insensitive to variations in $Y_{e}$ from the GR1D profile to neutrinoless $\beta$-equilibrium. All this leads us to the conclusion that it is thermal pressure support in the outer PNS core that is responsible for increasing the maximum stable gravitational PNS mass beyond that of a cold NS. Our finding is in agreement with the recent $\mathrm{BH}$ formation simulations of Sumiyoshi et al. (2007) and Fischer et al. (2009), who noted the same differences to cold TOV solutions, but did not pinpoint their precise cause. However, our result is in disagreement with Burrows (1988) who reported maximum PNS masses within a few percent of a solar mass off their cold-NS values. This could be related to Burrows's specific choice of EOS. As we discuss below, stiff nuclear EOS have a more limited response to thermal effects. Another resolution to this disagreement could be the nature of his PNS cooling simulations that were not hydrodynamic, but rather employed a Henyey relaxation approach with imposed accretion.

We find the same overall systematics of increased maximum PNS mass due to thermal pressure support for the entire set of progenitors evolved with the LS180, LS220, and HShen EOS (variations due to differences in progenitor structure are discussed in Section 4.4). In the sequence of the LS EOS, the relevance of thermal pressure support decreases with increasing 
stiffness. In the case of the perhaps unphysically stiff LS375 EOS, the effect of high temperatures in the outer PNS core is reversed: the PNSs in GR1D simulations become unstable to collapse at lower maximum masses than their cold counterparts. This very surprising observation is understood by considering that in GR, higher temperatures not only add thermal pressure support to the PNS, but also increase its mass energy. This results in a deeper effective potential well and, thus, is destabilizing. In the LS180, LS220, and HShen case, the added thermal pressure support is significant and dominates over the latter effect. In the very stiff LS375 EOS, the added thermal pressure component is negligible, and the destabilizing effect dominates.

Finally, we point out quantitative differences in models evolved with and without neutrino pressure in the dense neutrino-opaque core. In the $s 40 \mathrm{WH} 07$ model evolved with the HShen EOS, the difference in the maximum gravitational mass is $\sim 0.08 M_{\odot}(\sim 3 \%)$ and the difference in the time to $\mathrm{BH}$ formation is $\sim 160 \mathrm{~ms}(\sim 14 \%)$. These numbers depend on the employed EOS and progenitor model. In test calculations with a variety of progenitors and EOS, we generally find increases of the maximum PNS gravitational mass of $\lesssim 5 \%$.

\subsection{Influence of Presupernova Structure}

The failure of a CCSN becomes definite only when accretion pushes the PNS over its maximum mass and a $\mathrm{BH}$ forms. Hence, the time to $\mathrm{BH}$ formation is a hard upper limit to the time available for the supernova mechanism to reenergize the shock. We will demonstrate in this section that it is possible to estimate, for a given nuclear EOS, the postbounce time to $\mathrm{BH}$ formation in non- or slowly spinning on the basis of a single parameter, progenitor bounce compactness, $\xi_{2.5}$, which we introduced in Section 3. In Figure 6, we plot the postbounce time to $\mathrm{BH}$ formation $\left(t_{\mathrm{BH}}\right)$ as a function of $\xi_{2.5}$ for all nonrotating models considered in this study and listed in Table 2 . The distribution of data points for each EOS can be fit with a function $\propto\left(\xi_{2.5}\right)^{-3 / 2}$. This remarkable result can be understood as follows: using Kepler's third law, consider the Newtonian free fall time to the origin for a mass element $d m$ initially located at $r_{*}$ and on a radial orbit about a point mass of $M^{*} \gg d m$,

$$
t_{\mathrm{ff}}=\frac{1}{2} \sqrt{\frac{4 \pi^{2} a^{3}}{G M^{*}}}=\pi \sqrt{\frac{r_{*}^{3}}{8 G M^{*}}} .
$$

Here, for clarity, the quantities are in cgs units. $G$ is the gravitational constant and $a$ is the semimajor axis equal to half of the apoapsis, $r_{*}$. Recalling the definition of $\xi_{2.5}$, if the mass element $d m$ is located at a mass coordinate of $2.5 M_{\odot}$ and at a radial coordinate of $r_{*}$, then $r_{*}=2500 \mathrm{~km} / \xi_{2.5}$, and we can write the free fall time in terms of $\xi_{2.5}$,

$$
t_{\mathrm{ff}}^{2.5 M_{\odot}}=0.241\left(\xi_{2.5}\right)^{-3 / 2} \mathrm{~s}
$$

In Figure 6, we overplot this Newtonian free fall time for a mass element at baryonic mass coordinate $2.5 M_{\odot}$ as a function of $\xi_{2.5}$. For small $\xi_{2.5}$, the mass element begins its free fall from a large radius and, hence, takes longer to reach to origin. In general, material in outer layers of the star will not begin to fall freely until it loses pressure support. Hence, the free fall approximation is not exact (within a factor of $\sim 2$; Burrows 1986), but describes the general behavior of $t_{\mathrm{BH}}$ very well. The deviation of data points from the free fall curve is because the maximum PNS mass is different for each model and EOS. Models evolved with the LS180 EOS have PNSs with maximum

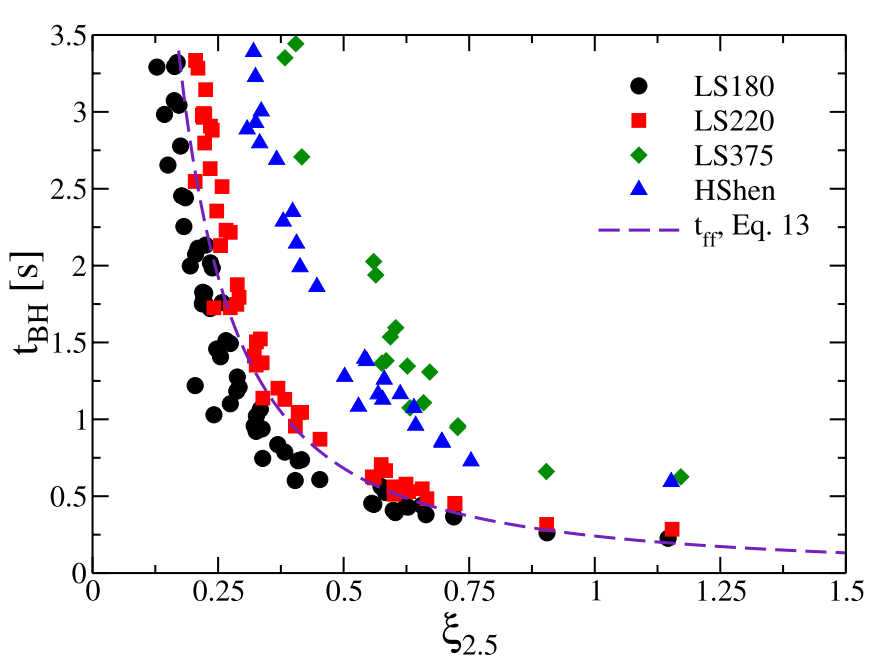

Figure 6. $\mathrm{BH}$ formation time as a function of the bounce compactness $\left(\xi_{2.5}\right)$ for all nonrotating models presented in Table 2 that form BHs within $3.5 \mathrm{~s}$ of bounce. Simulations performed with the LS180, LS220, LS375, and HShen EOS are labeled with circles, squares, diamonds, and triangles, respectively. Also shown (dashed line) is the free fall time to the origin (Equation (13)) of a mass element located at a baryonic mass coordinate of $2.5 M_{\odot}$.

(A color version of this figure is available in the online journal.)

baryonic masses ranging from 2.1 to $2.5 M_{\odot}$. Models with low $\xi_{2.5}$ correspond to the lower end of this mass range. For these models, $t_{\mathrm{BH}}$ can be somewhat less than the free fall time of the $2.5 M_{\odot}$ mass element, because less material is needed to form a $\mathrm{BH}$. The maximum baryonic PNS mass range for models using the LS220 EOS is somewhat higher, 2.3-2.6 $M_{\odot}$. BH formation times for these models are more in line with the Newtonian free fall prediction. Models evolved with the LS375 and HShen EOS have PNSs that must accrete upward of $\sim 3 M_{\odot}$ of material before becoming unstable. This significantly increases $t_{\mathrm{BH}}$ above the free fall prediction for the $\xi_{2.5}$ characteristic mass element.

Thermal pressure support can increase the maximum gravitational PNS mass $\left(M_{\mathrm{g}, \max }\right)$ as we have seen in Section 4.3 for the $s 40 \mathrm{WH} 07$ model. In Figure 7 , we plot $M_{\mathrm{g}, \max }$ as a function of $\xi_{2.5}$ for all nonrotating models listed in Table 2 . As obvious from this figure, $M_{\mathrm{g} \text {, max }}$ depends in a predictable way not only on the EOS, but also on the bounce compactness of the presupernova model. Progenitors with high $\xi_{2.5}$, in addition to forming BHs faster, create PNSs that are stable to higher masses. This is a simple consequence of the fact that progenitors with larger $\xi_{2.5}$ have iron cores with systematically higher entropies and masses significantly above the cold Chandrasekhar mass (see Table 1 and Baron \& Cooperstein 1990 and Burrows \& Lattimer 1983). Adiabatic collapse leads to higher PNS temperatures after bounce in progenitors with high $\xi_{2.5}$ and, hence, more thermal support. This leads to higher maximum PNS masses. This effect can be large, up to $25 \%$ for models with large $\xi_{2.5}$ and soft EOS.

\subsection{Preventing BH Formation with Artificial Neutrino-driven Explosions}

In a successful CCSN, the shock is reenergized before enough material can accrete onto the PNS to make it unstable. While fully self-consistent spherically symmetric simulations generally fail to explode in all but a few very low mass progenitors (cf. Kitaura et al. 2006; Burrows et al. 2007a), one can explode any star by the 1D neutrino mechanism by artificially increasing the energy deposition in the postshock region. Without such an increase, all of our simulations fail to 


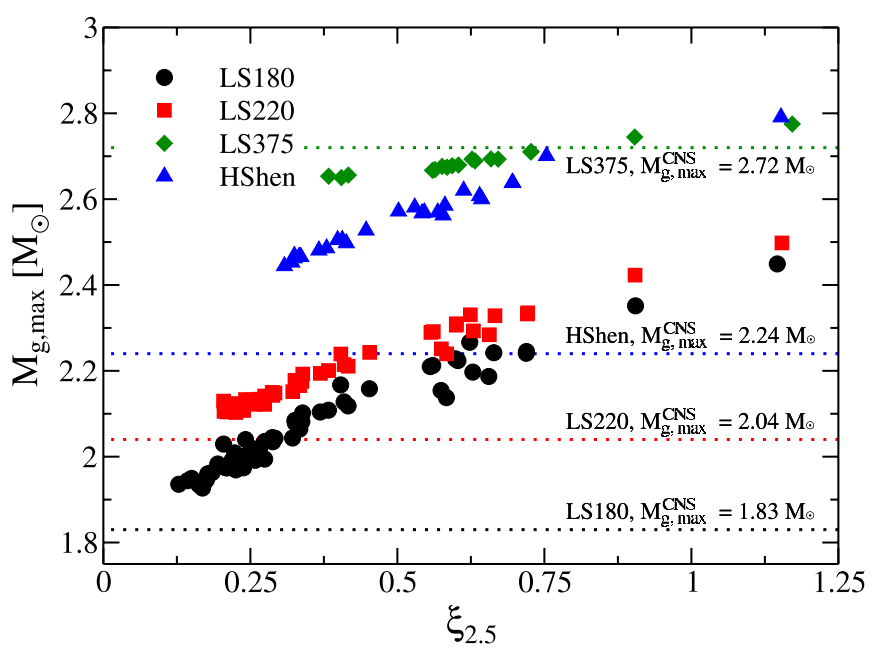

Figure 7. Maximum gravitational PNS masses as a function of the bounce compactness $\left(\xi_{2.5}\right)$ for all nonrotating models presented in Table 2 that form BHs within $3.5 \mathrm{~s}$ after bounce. Simulations performed with the LS180, LS220, LS375, and HShen EOS are labeled with circles, squares, diamonds, and triangles, respectively. Also shown (dotted lines, labeled) are the maximum gravitational cold-neutron star $(\mathrm{CNS})$ masses, $M_{\mathrm{g}, \max }^{\mathrm{CNS}}$, numerical values are $1.83,2.04,2.72$, and $2.24 M_{\odot}$ for the LS180, LS220, LS375, and HShen EOS, respectively.

(A color version of this figure is available in the online journal.)

explode. Our parameterized heating ( $f_{\text {heat }}$ in Equation 9 ) allows us to explore "how much" neutrino heating is needed to explode a given model (in 1D). By comparison with results from previous self-consistent radiation hydrodynamics simulations we can then estimate whether a given progenitor and EOS combination is more likely to lead to an explosion or $\mathrm{BH}$ formation.

Our method for driving explosions is similar to Murphy \& Burrows (2008), but has the advantage of being proportional to the neutrino luminosity obtained from the neutrino leakage scheme and therefore conserves energy. We iteratively determine the critical value of $f_{\text {heat }}$ to within $1 \%$ to what is needed for a successful explosion for a large subset of our models and the LS180, LS220, and HShen EOS. Of particular interest in this analysis is the time-averaged heating efficiency of the critical model, $\bar{\eta}_{\text {heat }}^{\text {crit }}$. We define $\bar{\eta}_{\text {heat }}$ as

$$
\bar{\eta}_{\text {heat }}=\overline{\int_{\text {gain }} \dot{q}_{\nu}^{+} d V /\left(L_{v_{e}}+L_{\bar{v}_{e}}\right)_{r_{\text {gain }}}},
$$

where $\dot{q}_{v}^{+}$is the net energy deposition rate and the neutrino luminosities are taken at the gain radius. We perform the time average between bounce and explosion, the latter time defined as when the postshock region assumes positive velocities and accretion onto the PNS ceases. $\bar{\eta}_{\text {heat }}^{\text {crit }}$ is a useful quantity because it characterizes how much of the available luminosity must be redeposited on average to explode a given progenitor. This is rather independent of transport scheme and code. For example, for the $15 M_{\odot}$ ZAMS solar-metallicity progenitor of Woosley $\&$ Weaver (1995) we find $\bar{\eta}_{\text {heat }}^{\text {crit }} \sim 0.13$. Buras et al. (2006b) who also artificially exploded this progenitor in 1D, though with much more sophisticated neutrino transport, find ${ }^{1}$ an average heating efficiency of $0.1-0.15$ which is consistent with our result. Note, however, that Marek \& Janka (2009) observed in the same progenitor the onset of a self-consistent neutrinodriven explosion in 2D at an average heating efficiency of $\sim 0.07$.

\footnotetext{
1 This we deduce from their Figure 28, bottom panel. Note that their $\delta_{t} E_{\mathrm{cool}}$
} includes all neutrinos, not just $v_{e}$ and $\bar{v}_{e}$.

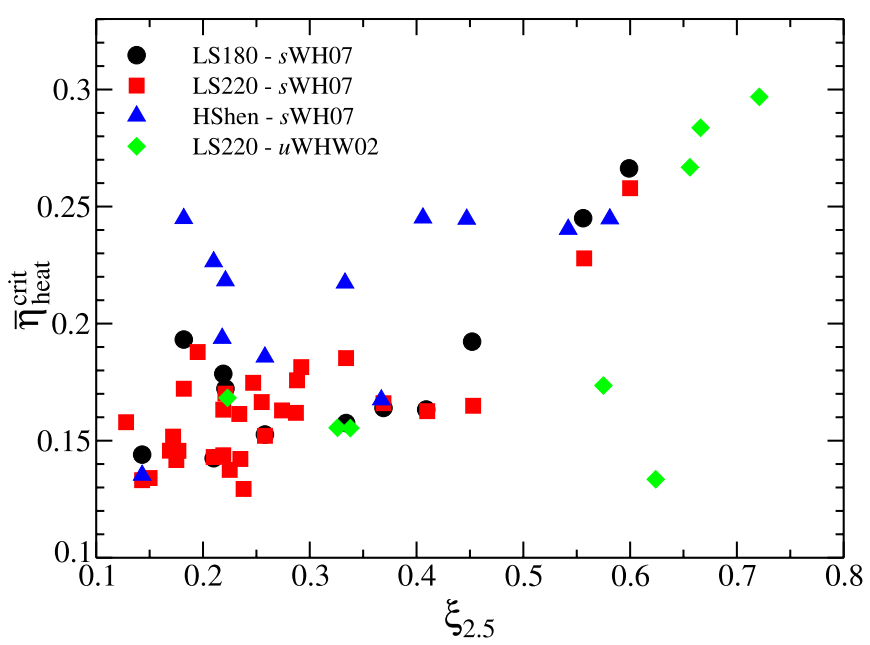

Figure 8. $\bar{\eta}_{\text {heat }}^{\text {crit }}$ obtained with GR1D as a function of bounce compactness. Plotted are models from the $s \mathrm{WH} 07$ data set using the LS180, LS220, and HShen EOS; and models from the $u$ WHW02 data set using the LS220 EOS.

(A color version of this figure is available in the online journal.)

This indicates a dependence of $\bar{\eta}_{\text {heat }}^{\text {crit }}$ on dimensionality, should be kept in mind, and is consistent with recent work that suggest that dimensionality may be the key to successful neutrino-driven explosions (Murphy \& Burrows 2008; Nordhaus et al. 2010).

Since GR1D's leakage/heating scheme is only a rough approximation to true neutrino transport, and because our simulations assume spherical symmetry, we cannot make very robust quantitative predictions for any one particular model, but rather study the collective trends exhibited by the entire set of 62 progenitors that we consider here. In Figure 8, as a function of bounce compactness $\xi_{2.5}$, we plot $\bar{\eta}_{\text {heat }}^{\text {crit }}$ for all considered models and EOS. The data are summarized in Table 4. We can divide the results into two general regimes: models with $\xi_{2.5} \lesssim 0.45$ and those with $\xi_{2.5} \gtrsim 0.45$.

For many models with $\xi_{2.5} \lesssim 0.45$, oscillations in the shock position are ubiquitous near the transition from failing to exploding supernovae in 1D (cf. Murphy \& Burrows 2008; Buras et al. 2006b; Fernández \& Thompson 2009). For both the LS180 and LS220 EOS, the $\bar{\eta}_{\text {heat }}^{\text {crit }}$ required for an explosion, modulo noise, is roughly constant and $\sim 0.16$ on average for low $\xi_{2.5}$ models. Hence, explosion is the likely outcome of core collapse for progenitors with $\xi_{2.5} \lesssim 0.45$ if the nuclear EOS is similar to the LS180 or LS220 case.

The noise in the $\bar{\eta}_{\text {heat }}^{\text {crit }}$ distribution (absolute variations by up to $\sim 10 \%$ ) is in part a consequence of variations in postbounce dynamics, such as the number and duration of pre-explosion oscillations. Compositional interfaces in some progenitor models, where jumps in the density lead to jumps in the accretion rate, also affect individual models leading to variations in $\bar{\eta}_{\text {heat }}^{\text {crit }}$. For the LS180 and LS220 EOS, any differences in $\bar{\eta}_{\text {heat }}^{\text {crit }}$ with choice of EOS are indistinguishable given the noise in the data.

For progenitors with $\xi_{2.5} \gtrsim 0.45$, the $\bar{\eta}_{\text {heat }}$ required to cause an explosion increases with $\xi_{2.5}$ when run with the LS180 or LS220 EOS. Progenitors in this regime have tremendous postbounce accretion rates, accumulating $\gtrsim 2 M_{\odot}$ of baryonic material behind the shock within the first $\sim 200 \mathrm{~ms}$ after bounce. Without explosion, they form BHs within $\lesssim 0.8 \mathrm{~s}$ (with the LS180 and LS220 EOS). Hence, a very high heating efficiency of $\bar{\eta}_{\text {heat }} \gtrsim 0.23-0.27$ is necessary to drive an explosion at early times against the huge ram pressure of accretion. It appears unlikely, even when multi-dimensional dynamics are factored in, that progenitors with $\xi_{2.5} \gtrsim 0.45$ can be exploded via the 
Table 4

Explosion Properties

\begin{tabular}{|c|c|c|c|c|c|c|c|}
\hline $\begin{array}{c}M_{\text {ZAMS }} \\
\left(M_{\odot}\right) \\
\end{array}$ & $\xi_{2.5}$ & $f_{\text {heat }}^{\text {crit }}$ & $\bar{\eta}_{\text {heat }}^{\text {crit }}$ & $\begin{array}{c}M_{\text {ZAMS }} \\
\left(M_{\odot}\right) \\
\end{array}$ & $\xi_{2.5}$ & $f_{\text {heat }}^{\text {crit }}$ & $\bar{\eta}_{\text {heat }}^{\text {crit }}$ \\
\hline \multicolumn{4}{|c|}{$s \mathrm{WH} 07$ LS220 } & \multicolumn{4}{|c|}{$s$ WH07 LS180 } \\
\hline 14 & 0.128 & 1.17 & 0.158 & 15 & 0.182 & 1.16 & 0.193 \\
\hline 15 & 0.182 & 1.17 & 0.172 & 21 & 0.143 & 1.32 & 0.144 \\
\hline 16 & 0.150 & 1.33 & 0.134 & 23 & 0.452 & 1.18 & 0.192 \\
\hline 17 & 0.169 & 1.32 & 0.146 & 24 & 0.409 & 1.16 & 0.163 \\
\hline 18 & 0.195 & 1.17 & 0.188 & 25 & 0.334 & 1.13 & 0.158 \\
\hline 19 & 0.177 & 1.24 & 0.146 & 27 & 0.258 & 1.18 & 0.153 \\
\hline 20 & 0.288 & 1.15 & 0.176 & 30 & 0.219 & 1.16 & 0.179 \\
\hline 21 & 0.143 & 1.34 & 0.133 & 35 & 0.369 & 1.14 & 0.164 \\
\hline 22 & 0.292 & 1.15 & 0.181 & 40 & 0.599 & 1.32 & 0.266 \\
\hline 23 & 0.453 & 1.17 & 0.165 & 45 & 0.556 & 1.26 & 0.245 \\
\hline 24 & 0.410 & 1.15 & 0.163 & 50 & 0.221 & 1.18 & 0.172 \\
\hline 25 & 0.334 & 1.14 & 0.185 & 80 & 0.210 & 1.22 & 0.142 \\
\hline 26 & 0.235 & 1.21 & 0.142 & \multicolumn{4}{|c|}{ sWH07 HShen } \\
\hline 27 & 0.258 & 1.20 & 0.152 & 15 & 0.182 & 1.30 & 0.245 \\
\hline 28 & 0.274 & 1.16 & 0.163 & 21 & 0.143 & 1.50 & 0.135 \\
\hline 29 & 0.225 & 1.25 & 0.138 & 23 & 0.447 & 1.27 & 0.245 \\
\hline 30 & 0.219 & 1.18 & 0.163 & 24 & 0.406 & 1.31 & 0.245 \\
\hline 31 & 0.219 & 1.21 & 0.144 & 25 & 0.333 & 1.49 & 0.217 \\
\hline 32 & 0.255 & 1.17 & 0.166 & 27 & 0.258 & 1.52 & 0.186 \\
\hline 33 & 0.287 & 1.15 & 0.162 & 30 & 0.218 & 1.32 & 0.194 \\
\hline 35 & 0.369 & 1.13 & 0.166 & 35 & 0.367 & 1.37 & 0.167 \\
\hline 40 & 0.600 & 1.30 & 0.259 & 40 & 0.581 & 1.22 & 0.245 \\
\hline 45 & 0.557 & 1.25 & 0.228 & 45 & 0.542 & 1.24 & 0.240 \\
\hline 50 & 0.221 & 1.19 & 0.170 & 50 & 0.221 & 1.41 & 0.218 \\
\hline 55 & 0.238 & 1.24 & 0.129 & 80 & 0.210 & 1.50 & 0.226 \\
\hline 60 & 0.175 & 1.29 & 0.142 & & & & \\
\hline 70 & 0.234 & 1.21 & 0.161 & \multicolumn{4}{|c|}{ sWW95 LS180 } \\
\hline 80 & 0.210 & 1.24 & 0.143 & 15 & 0.088 & 1.33 & 0.130 \\
\hline 100 & 0.247 & 1.15 & 0.175 & & & & \\
\hline 120 & 0.172 & 1.25 & 0.152 & & & & \\
\hline \multicolumn{8}{|c|}{$u$ WHW02 LS220 } \\
\hline 20 & 0.338 & 1.13 & 0.155 & 40 & 0.721 & 1.44 & 0.297 \\
\hline 25 & 0.223 & 1.16 & 0.168 & 45 & 0.656 & 1.22 & 0.267 \\
\hline 30 & 0.326 & 1.13 & 0.156 & 50 & 0.575 & 1.09 & 0.174 \\
\hline 35 & 0.666 & 1.37 & 0.284 & 60 & 0.624 & 1.12 & 0.133 \\
\hline
\end{tabular}

Notes. $f_{\text {heat }}^{\text {crit }}$ corresponds to the critical value needed to cause a successful explosion in GR1D. $\bar{\eta}_{\text {heat }}^{\text {crit }}$ is the associated critical average heating efficiency defined in Equation (14).

neutrino mechanism. The most likely outcome of core collapse in such stars is BH formation.

We draw the reader's attention to two outliers in the $u$ WHW02 data set included in Figure 8, the $u 50$ WHW02 and $u 60$ WHW02 progenitors. These models have high $\xi_{2.5}$, but feature compositional interfaces where the density drops by $\sim 50 \%$. These are located at a mass coordinate of $1.82 M_{\odot}$ and $2.22 M_{\odot}$ in $u 50$ WHW02 and $u 60$ WHW02, respectively. When such an interface advects through the shock, the accretion rate drops suddenly, but the core neutrino luminosity remains large and an explosion is immediately launched. This results in a small value of $f_{\text {heat }}^{\text {crit }}$ and, therefore, in a low required $\bar{\eta}_{\text {heat }}$. This demonstrates that the single parameter $\xi_{2.5}$ is not always sufficient to predict a progenitor's fate.

In models with $\xi_{2.5} \lesssim 0.45$ and calculated using the HShen EOS, both $\bar{\eta}_{\text {heat }}^{\text {crit }}$ and $f_{\text {heat }}^{\text {crit }}$ are systematically higher than with the LS180 and LS220 EOS and explosion is less likely. Furthermore, the qualitative behavior of our simulations is different with the HShen EOS. In many models with subcritical $f_{\text {heat }}$ and $\bar{\eta}_{\text {heat }}$, the shock is revived and begins to propagate to large radii of $\mathcal{O}\left(10^{3}-10^{4} \mathrm{~km}\right)$, but the material behind it fails to achieve positive velocities. Hence, accretion onto the PNS is slowed but does not cease. High values of $f_{\text {heat }}$ are needed to avoid this and achieve full explosions. We caution the reader that this regime may not be well modeled by our neutrino treatment. Nevertheless, our results suggest that systematically higher $f_{\text {heat }}^{\text {crit }}$ and $\bar{\eta}_{\text {heat }}^{\text {crit }}$ are required to explode models with the HShen EOS, even at low $\xi_{2.5}$. In contrast to models using the LS180 or LS220 EOS, models with $\xi_{2.5} \gtrsim 0.45$ with the HShen EOS require roughly constant $\bar{\eta}_{\text {heat }}$ to explode. Since the HShen EOS can support a high maximum mass, the PNS can withstand $\mathrm{BH}$ formation longer and explosions may set in at later postbounce times when the accretion rate has dropped sufficiently.

Finally, as an interesting aside, we point out the evolution of the $u 75 \mathrm{WHW} 02$ progenitor evolved with the LS220 EOS. This model has a bounce compactness of $\sim 1.15$ and, in the absence of an explosion, forms a BH $\sim 0.285 \mathrm{~s}$ after bounce (with the LS220 EOS). This progenitor has a compositional interface at which the density drops by $\sim 50 \%$ that is located at a baryonic mass coordinate of $\sim 2.5 M_{\odot}$. This is very close to the maximum mass of the $u 75 \mathrm{WHW} 22$ PNS (with the LS220 EOS) and well above the maximum cold NS (baryonic) mass. The model can be made to explode with $f_{\text {heat }}^{\text {crit }}=1.35$ with a corresponding $\bar{\eta}_{\text {heat }}^{\text {crit }}=0.287$. The resulting PNS has a baryonic (gravitational) mass of $\sim 2.54 M_{\odot}\left(2.44 M_{\odot}\right)$. Interestingly, within $\sim 100 \mathrm{~ms}$ after the launch of the explosion, cooling of the outer PNS layers removes sufficient thermal pressure, rendering the PNS unstable to collapse and $\mathrm{BH}$ formation. This scenario will necessarily occur within the cooling phase for any PNS that is initially thermally supported above the maximum cold NS baryonic mass and is another avenue to $\mathrm{BH}$ formation. In our simulations, this condition is also met only in very few other models with very high $\xi_{2.5}$ and fairly soft EOS, such as the 23, 40, and $45 M_{\odot}$ progenitors from the $s \mathrm{WH} 07$ series using the LS180 EOS. In order to fully investigate this BH formation channel, a more sophisticated neutrino treatment is required that allows accurate long-term modeling of PNS cooling (Pons et al. 1999), since, in general, the Kelvin-Helmholtz cooling phase of PNS is $\mathcal{O}(10-100 \mathrm{~s})$.

\subsection{Connection to Stellar Evolution and ZAMS Conditions}

\subsubsection{ZAMS Mass and Metallicity}

Having established the systematic dependence of core collapse and $\mathrm{BH}$ formation on progenitor bounce compactness in Section 4.4, we now go further and attempt to connect to the conditions at ZAMS. Doing this is difficult, and, given the current state and limitations of stellar evolution theory and modeling, can be done only approximately. In general, presupernova stellar structure will depend not only on initial conditions at ZAMS (mass, metallicity, rotation), but also on particular evolution history and physics (binary effects, [rotational] mixing, magnetic fields, nuclear reaction rates, and mass loss; cf. Woosley et al. 2002). While keeping this in mind, we limit ourselves in the following to the exploration of single-star, nonrotating progenitors without magnetic fields. We focus on collapse models run with the LS220 EOS, but the general trends with EOS observed in the previous sections extend to here.

In the top panel of Figure 9, we plot the bounce compactness $\xi_{2.5}$ as a function of progenitor ZAMS mass $M_{\text {ZAMS }}$ for a range of progenitors from multiple stellar evolutionary studies. Even within a given model set, the $M_{\text {ZAMS }}-\xi_{2.5}$ mapping is highly 


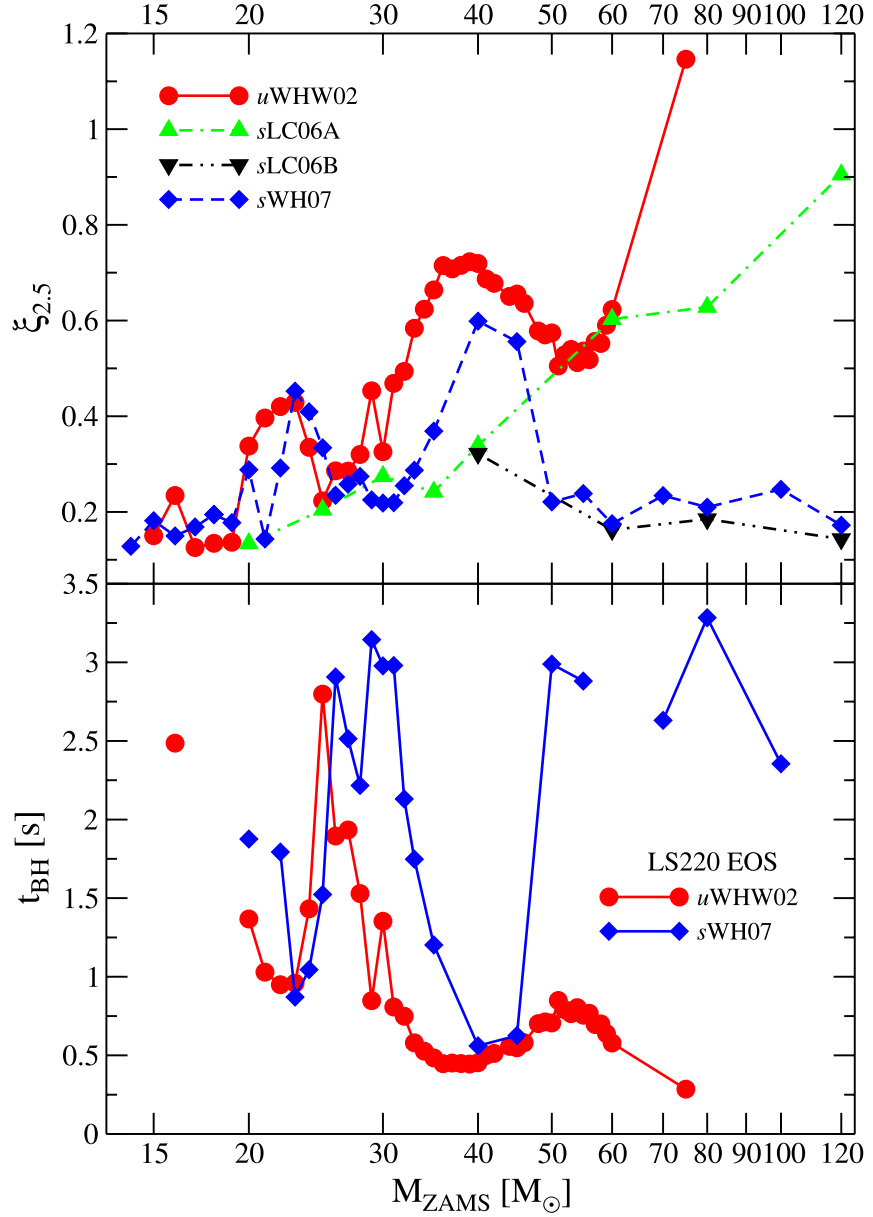

Figure 9. Bounce compactness (top panel) and time to $\mathrm{BH}$ formation (bottom panel) as functions of ZAMS mass for various progenitor sets. $\xi_{2.5}$ is determined for each model at bounce using Equation (10). $t_{\mathrm{BH}}$ for each model is obtained using the LS220 EOS and assuming no explosion. The times to $\mathrm{BH}$ formation for progenitor models that take longer than $3.5 \mathrm{~s}$ to form a $\mathrm{BH}$ are not shown. Breaks in the lines connecting models indicate this. For clarity, the time to $\mathrm{BH}$ formation is not shown for the $s \mathrm{LC} 06 \mathrm{~A} / \mathrm{B}$ series, but is provided in Table 2.

(A color version of this figure is available in the online journal.)

non-monotonic. At the low end of $M_{\text {ZAMs }}$ covered by Figure 9 , where mass loss has little influence even in progenitors of solar metallicity, variations in $\xi_{2.5}$ are due predominantly to particularities in late burning stages, caused, e.g., by convective versus radiative core burning and/or differences in shell burning episodes (Woosley et al. 2002). At the high ZAMS-mass end, $\xi_{2.5}$ is determined by a competition of mass loss and rapidity of nuclear-burning evolution.

The bottom panel of Figure 9 depicts the time to $\mathrm{BH}$ formation $t_{\mathrm{BH}}$ in a failing CCSN as a function of $M_{\text {ZAMS }}$ for the $s \mathrm{WH} 07$ solar-metallicity progenitors of Woosley \& Heger (2007) and the $u$ WHW02 $10^{-4}$ solar-metallicity models of Woosley et al. (2002). Models of very low $\xi_{2.5}$ that require more than $3.5 \mathrm{~s}$ to make a $\mathrm{BH}$ are omitted. As demonstrated in Section 4.4, $t_{\mathrm{BH}}$ scales $\propto\left(\xi_{2.5}\right)^{-3 / 2}$ and, hence, progenitors that form $\mathrm{BH}$ the fastest and are (generally, cf. Section 4.5) the hardest to explode are those with high values of $\xi_{2.5}$. In the lowmetallicity $u$ WHW02 series whose progenitors experience only minuscule mass loss, BHs form within $\lesssim 1 \mathrm{~s}$ of bounce for $M_{\text {ZAMS }} \gtrsim 30 M_{\odot}$ and the high bounce compactness $\xi_{2.5} \gtrsim 0.45$ makes a successful shock revival rather unlikely (Section 4.5). Hence, the most likely outcome of core collapse is $\mathrm{BH}$ formation in these progenitors. This may also be the case for $u$ WHW02 progenitors in the ZAMS mass range from $\sim 20$ to $25 M_{\odot}$. The $s \mathrm{WH} 07$ progenitors have high $\xi_{2.5}$ and form BHs rapidly only in the $M_{\text {ZAMS }}$ ranges $\sim 23-25 M_{\odot}$ and $\sim 35-45 M_{\odot}$. At higher ZAMS masses, strong $O$-star mass loss leads to an early removal of the hydrogen envelope. Subsequent mass loss in the W-R phase leads to bare, low-mass, low-compactness carbon-oxygen cores in the most massive progenitors that are unlikely to make BHs.

\subsubsection{Variations with Mass-loss Prescriptions}

Mass loss is key in determining the observational appearance of a successful CCSN (e.g., Filippenko 1997; Smith et al. 2010), but, as we have seen in Section 4.6.1, also has a strong effect on presupernova core structure and, thus, on the outcome of core collapse. The details of mass loss in massive stars are still rather uncertain (e.g., Smith et al. 2010), and, unfortunately, there are few stellar evolution studies that have studied the effects of variations in mass-loss prescriptions. Limongi \& Chieffi $(2006)^{2}$ performed such a study, adopting two different mass-loss rates for the $\mathrm{W}-\mathrm{R}$ stage of solar-metallicity stars with $M \gtrsim 40 M_{\odot}$. The $s \mathrm{LC} 06 \mathrm{~B}$ models are evolved with the W-R mass-loss rates of Langer (1989) that are similar to those used in the $s$ WH07 set of Woosley \& Heger (2007). As depicted in the top panel of Figure 9, high-mass $s$ LC06B and $s$ WH07 models have similar low $\xi_{2.5}$ and most likely do not form BHs but rather explode as type-Ibc CCSNe. The models of the sLC06A set were evolved with the lower (factor of $\sim 2$ ) W-R mass-loss rates of Nugis \& Lamers (2000). The $s$ LC06A 60, 80, and $120 M_{\odot}$ progenitors have much more mass left at the presupernova stage $\left(M_{\text {pre-SN }} \sim 17-30 M_{\odot}\right.$, Figure 2$)$ and very high bounce compactness of $\xi_{2.5} \sim 0.6-0.9$. In the likely case of CCSN failure, a BH forms within $\sim 0.5 \mathrm{~s}$ with the LS180 EOS and within $\sim 1.5$ s for all other EOS.

The above results highlight the sensitivity of outcome predictions on mass-loss physics and a more solid understanding of this key ingredient will be necessary to robustly connect ZAMS masses to the outcome of core collapse for massive stars around and above solar metallicity.

\subsection{The Formation of Rotating Black Holes}

Rotation, if sufficiently rapid, alters the CCSN dynamics via centrifugal support. This important effect is captured by GR1D's 1.5D rotation treatment, albeit approximately. Initially, centrifugal support acts to slow the collapse of the inner core, delaying core bounce. At bounce, lower peak densities are reached, the hydrodynamic shock forms at a larger radius, and its enclosed mass is larger. Conservation of angular momentum spins up the core from precollapse angular velocities that may be of order rad s $\mathrm{s}^{-1}$ to $\mathcal{O}\left(1000 \mathrm{rad} \mathrm{s}^{-1}\right)$ as the core, initially with $r \sim \mathcal{O}(1000 \mathrm{~km})$, collapses to a PNS with $r \sim \mathcal{O}(30 \mathrm{~km})$. During the postbounce evolution, the spinning PNS is stabilized at lower densities, is less compact, generally colder, and has a softer neutrino spectrum than a non-spinning counterpart (Ott et al. 2008, and references therein).

\subsubsection{Models with Parameterized Rotation}

We investigate the effect of rotation in failing CCSNe by assigning specific angular momentum profiles to the $u$ WHW02 model set (see Section 3) via Equation (11). This rotation

\footnotetext{
2 See also Limongi \& Chieffi (2009) and Meynet \& Maeder (2003).
} 


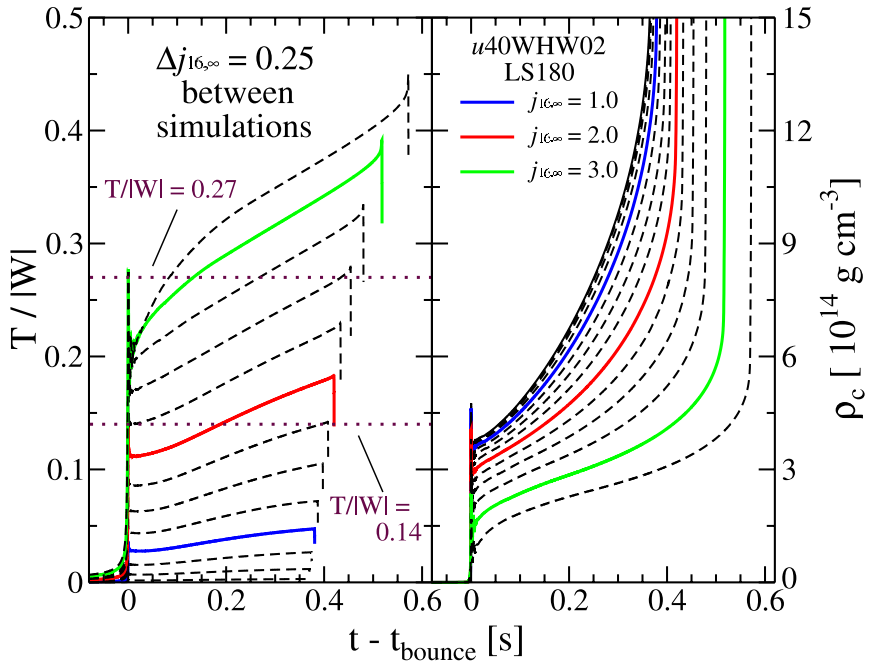

Figure 10. $T /|W|$ (left) and central density $\left(\rho_{c}\right)$ (right) during the postbounce evolution of the $u 40$ WHW02 model using the LS180 EOS and 14 different initial specific angular momentum profiles. We vary $j_{16, \infty}$ from 0 to 3.25 in increments of 0.25 . For clarity we highlight with solid lines the simulations with integer values of $j_{16, \infty}$. Lines at $T /|W|=0.27$ and 0.14 are added to denote the dynamical and secular rotational instability thresholds.

(A color version of this figure is available in the online journal.)

law approximates what is generally found in stellar evolution calculations that account for rotation (Heger et al. 2000; see Ott et al. 2006 for comparison plots). The inner iron core $\left(\sim 1 M_{\odot}\right)$ is rotating almost uniformly. Outside of this core, the angular velocity drops roughly $\propto r^{-2}$. In Table 5 , we summarize key parameters of our rotating model set. Among them is $T /|W|$, the ratio of rotational kinetic energy to gravitational binding energy. It is particularly indicative of the dynamical relevance of rotation.

In the right panel of Figure 10, we plot the central density evolution of model $u 40$ WHW02 run with the LS180 EOS for $j_{16, \infty}$ ranging from 0 to 3.25 in increments of 0.25 . While we choose the $u 40$ WHW02 model here, the results are generic and apply to all progenitors. $A_{M_{\odot}}$, of Equation (11), is $936 \mathrm{~km}$ for this model and the initial central rotation rate is $1.14 \times j_{16, \infty} \mathrm{rad} \mathrm{s}^{-1}$. The nonrotating model takes $\sim 433 \mathrm{~ms}$ to reach bounce and a further $\sim 369 \mathrm{~ms}$ before the PNS becomes unstable to collapse to a BH with a gravitational mass of $2.24 M_{\odot}$. For the $j_{16, \infty}=1,2$, and 3 models, respectively, the times to bounce are $11 \mathrm{~ms}, 47 \mathrm{~ms}$, and $125 \mathrm{~ms}$ greater than in the nonrotating case. Their times to $\mathrm{BH}$ formation $t_{\mathrm{BH}}$ are $12 \mathrm{~ms}, 52 \mathrm{~ms}$, and $150 \mathrm{~ms}$ longer than in the nonrotating case. The maximum gravitational PNS masses $M_{\mathrm{g}, \max }$ are $0.03 M_{\odot}, 0.09 M_{\odot}$, and $0.28 M_{\odot}$ greater. We find that the time to bounce, time to $\mathrm{BH}$ formation, and maximum gravitational PNS mass increase above the nonrotating values proportional to $\sim\left(j_{16, \infty}\right)^{2}$. The increase in $t_{\mathrm{BH}}$ is due almost entirely to the increase in $M_{g}$, max , since the accretion rate is not significantly affected by rotation.

The lower temperatures and densities of rotating PNSs lead to systematically lower mean neutrino energies and total radiated energy from the PNS core (time-averaged total luminosities are summarized in Table 5). Fryer \& Heger (2000) and Ott et al. (2008), who considered similarly rapidly rotating models, also see this effect. There is a clear trend toward lower $L_{v}$ with increasing $j_{16, \infty}$ and for a given model and at a given time, with increasing $j_{16, \infty}$, less gravitational binding energy has been carried away by neutrinos and $M_{g}$ is larger. Given essentially unaltered accretion rates, one may expect earlier PNS collapse and BH formation. This, however, is not the case in models run with the LS180, LS220, and HShen EOS, since the centrifugally increased $M_{g \text {,max }}$ systematically outweighs the increased gravitational mass due to the lowered neutrino emission. For these EOS, the time to $\mathrm{BH}$ formation is delayed by rotation. For models run with the extremely stiff LS357 EOS the situation is different. For them, the centrifugal support provided by rotation is too weak to significantly enhance $M_{g}$, max and, hence, BHs form faster with increasing $j_{16, \infty}$.

In the left panel of Figure 10, we plot the $T /|W|$ evolution for the rotating $u 40$ WHW02 model series run with the LS180 EOS. During collapse, gravitational binding energy is transferred to rotational energy, increasing the value of $T /|W|$. Similar to how the central density overshoots its new equilibrium, $T /|W|$ also exhibits a local maximum at bounce. Continued accretion and contraction of the PNS increases $T /|W|$ throughout the postbounce evolution for all models. Initially very rapidly spinning models experience core bounce under the strong influence of centrifugal effects, leading to reduced compactness and $T /|W|$ at bounce. These qualitative features are in good agreement with what was found by previous extensive parameter studies of rotating core collapse (Ott et al. 2006; Dimmelmeier et al. 2008). Quantitatively, we find and summarize in Table 5 that models with $j_{16, \infty} \lesssim 1.5$ yield $T /|W| \lesssim 0.14$ throughout their entire evolution. Models with $j_{16, \infty} \gtrsim 2.25$ have $T /|W| \gtrsim$ 0.14 during their entire postbounce evolution. Models that have $j_{16, \infty} \lesssim 2.25$ have $T /|W| \lesssim 0.27$ at all times. Models with $j_{16, \infty} \gtrsim 2.5$ reach $T /|W| \gtrsim 0.27$ before $\mathrm{BH}$ formation. When considering these numbers, it is important to keep in mind that GR1D's $1.5 \mathrm{D}$ approach to rotation has the tendency to overestimate $T /|W|$ in rapidly spinning models. Ott et al. (2006) found model-dependent differences in $T /|W|$ of $\mathcal{O}(10 \%)$ between 1.5D and 2D. In addition, GR1D's neutrino leakage scheme also tends to lead to somewhat more compact PNS cores and consequently higher $T /|W|$ than would be expected from full neutrino transport calculations.

The systematics of $T /|W|$ depicted by Figure 10 (left) and listed in Table 5, albeit only approximate due to GR1D's 1.5D treatment of rotation, shed interesting light on the potential role of nonaxisymmetric rotational instabilities during the evolution of failing CCSNe. Of course, due to its 1.5D nature, GR1D cannot track the development of such multi-dimensional dynamics. Analytic theory and to some extent 3D computational modeling have identified multiple instabilities that may lead to triaxial deformation of PNSs, redistribution of angular momentum, and to the radiation of rotational energy and angular momentum via gravitational waves (see Stergioulas 2003 and Ott 2009 for reviews). A global dynamical instability sets in for $T /|W| \gtrsim$ 0.27 (Chandrasekhar 1969), leading to a lowest-order $m=2$ "bar" deformation. Global secular instability, driven by viscosity or GW back-reaction sets in at $T /|W| \gtrsim 0.14$ (Chandrasekhar 1970; Friedman \& Schutz 1978). Finally, dynamical shear instabilities, arising as a result of differential rotation, may lead to partial or global nonaxisymmetric deformation at even lower values of $T /|W|$ ( $\gtrsim 0.05$; e.g., Saijo et al. 2003; Ott et al. 2007; Scheidegger et al. 2008; Corvino et al. 2010, and references therein). In nature, and in full 3D simulations, these instabilities, through gravitational radiation or redistribution of angular momentum, will effectively and robustly prevent $T /|W|$ from surpassing the corresponding $T /|W|$ threshold. The growth times of dynamical instabilities are short, $\mathcal{O}(\mathrm{ms})$. Secular instabilities grow on timescales set by the driving process and 
Table 5

Properties of Rotating Models

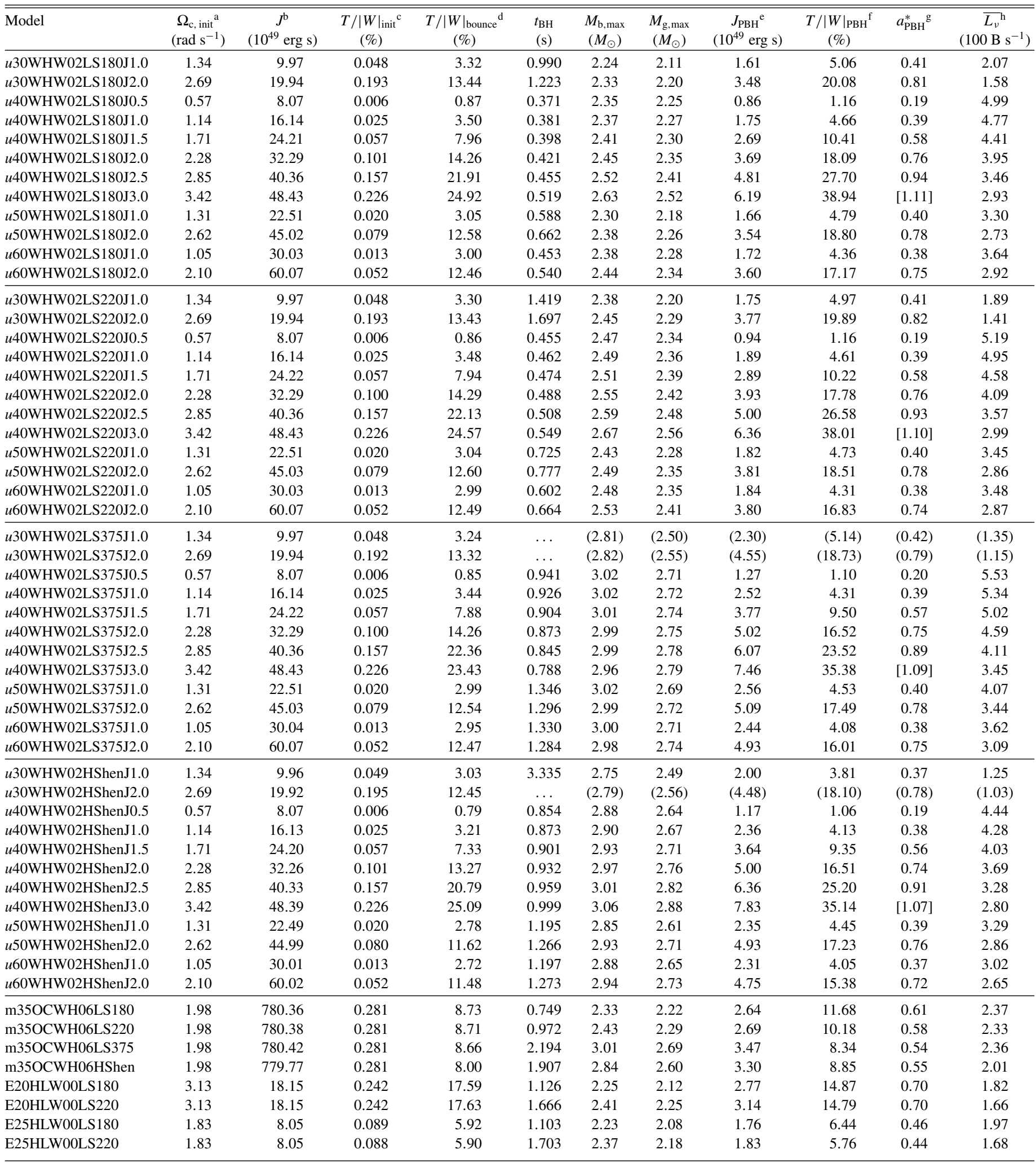

Notes. The $u$ series of presupernova models in this table are taken from the $Z_{\odot}=10^{-4}$ model set of Woosley et al. (2002). We imposed a rotation law via Equation (11), the value of $j_{16, \infty}$ is given in the model name following the letter J. The m35OC presupernova model is taken from Woosley \& Heger (2006) and both the E20 and E25 models are from Heger et al. (2000), these model are evolved with rotation. In simulations where a BH did not form within $3.5 \mathrm{~s}$ we give, in parenthesis, the values at this time.

${ }^{a}$ Initial central angular velocity of star. ${ }^{\mathrm{b}}$ Total angular momentum of star. ${ }^{\mathrm{c}}$ Initial $T /|W|$ of the star. ${ }^{\mathrm{d}} T /|W|$ of the star at bounce. ${ }^{\mathrm{e}}$ Angular momentum of protoblack hole (PBH) when $\alpha_{c}=0.3 .{ }^{\mathrm{f}} T /|W|$ of the star when $\alpha_{c}=0.3{ }^{\mathrm{g}}$ Dimensionless spin of the PBH when $\alpha_{c}=0.3$. Unphysical values for a BH are shown in braces [ $\cdots$ ].

${ }^{\mathrm{h}}$ Total neutrino luminosity averaged over postbounce time. 


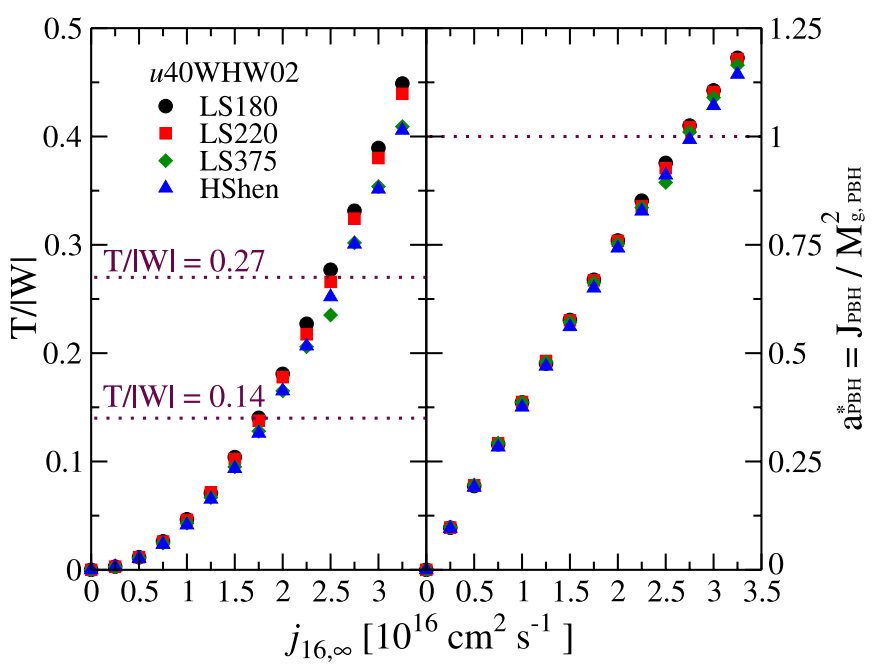

Figure 11. Left: $T /|W|$ for a range of initial $j_{16, \infty}$ and EOS for the $u 40$ WHW02 progenitor. We denote the value of $T /|W|$ thresholds for the dynamical rotational instability, $T /|W|_{\text {dyn }}=0.27$, and the secular instability, $T /|W|_{\mathrm{sec}}=0.14$. Right: dimensionless spin parameter $a_{\mathrm{PBH}}^{*}$ for the PNS at the last stable configuration prior to collapse to a $\mathrm{BH}$. Note that $a_{\mathrm{PBH}}^{*}>1$ is generally allowed by GR but a BH must have $a^{*}<1$. PNSs that could reach $a_{\mathrm{PBH}}^{*}>1$ are nonaxisymmetrically unstable and will be limited to $a_{\mathrm{PBH}}^{*}$ below 1. For the $u \mathrm{WHW} 02$ model, the initial central rotation rate is given as $\Omega_{c}=1.141 \times j_{16, \infty} \mathrm{rad} \mathrm{s}^{-1}$.

(A color version of this figure is available in the online journal.)

are typically $\mathcal{O}(\mathrm{s})$ (Lai \& Shapiro 1995). The low- $T /|W|$ shear instabilities in PNSs appear to grow on intermediate timescales of $\mathcal{O}(10-100 \mathrm{~ms})$ (e.g., Ott et al. 2007; Scheidegger et al. 2008).

In Figure 11, we plot the value of $T /|W|$ (left panel) and the dimensionless spin of the protoblack hole $(\mathrm{PBH}), a_{\mathrm{PBH}}^{*}=$ $J_{\mathrm{PBH}} /\left(M_{\mathrm{g}, \mathrm{PBH}}^{2}\right)$ (right panel) at the onset of BH formation (when $\left.\alpha_{c}=0.3\right)$ for the same values of $j_{16, \infty}$ used in Figure 10. Assuming that the entire PNS is promptly swallowed once the horizon appears, $a_{\mathrm{PBH}}^{*}$ corresponds to the $\mathrm{BH}$ birth spin. ${ }^{3}$ We again show results for model $u 40 \mathrm{WHW} 02$, but for all four EOS. The data are also presented in Table 5 for these and other models. $T /|W|$ at BH formation scales $\propto\left(j_{16, \infty}\right)^{2}: T /|W|_{\mathrm{PBH}}$ is $\sim 0.05, \sim 0.1, \sim 0.2$, and $\sim 0.3$ at $j_{16, \infty}$ of $\sim 1, \sim 1.5, \sim 2.2$, and $\sim 2.75$, respectively. $a_{\mathrm{PBH}}^{*}$ scales linearly with $j_{16, \infty}$, reaching a maximally Kerr value of $a_{\mathrm{PBH}}^{*} \sim 1$ at $j_{16, \infty} \sim 2.75 . T /|W|_{\mathrm{PBH}}$ and $a_{\mathrm{PBH}}^{*}$ vary little with EOS.

A disturbing fact depicted by Figure 11 is that our 1.5D simulations predict $\mathrm{BH}$ birth spins $a^{*} \gtrsim 1$ for $j_{16, \infty} \gtrsim 2.75$. In Kerr theory, such BHs cannot exist with a horizon. They would instead be naked singularities, violating the cosmic censorship conjecture (Penrose 1969). However, when comparing right and left panels of Figure 11, one notes that all models achieving $a^{*} \gtrsim 1$ are predicted to reach $T /|W|$ in excess of 0.27 . Hence, in nature and in a 3D simulation, these PNS will be dynamically nonaxisymmetrically unstable and angular momentum redistribution and gravitational radiation will limit their $T /|W|$ robustly below $\sim 0.27$, corresponding to $a^{*} \lesssim 0.9$. Rotational instabilities at lower values of $T /|W|$ may also be relevant. Dynamical shear instabilities have timescales significantly less than the time to $\mathrm{BH}$ formation. Secular rotational instabilities may be relevant if the true nuclear EOS allows for a large maximum

\footnotetext{
3 Note that this may not necessarily be what happens. Outer PNS material may become centrifugally supported, falling into the nascent $\mathrm{BH}$ only on an accretion timescale (Duez et al. 2006).
}

PNS mass as more time is needed to accrete the necessary material to form a BH (see Section 4.3). Large $t_{\mathrm{BH}}$ is also possible if $\xi_{2.5}$ is small (see Section 4.4) therefore allowing secular instabilities to grow. In all rotating models considered here (see Table 5), PNSs stable against the dynamical rotational instability with $T /|W| \lesssim 0.25-0.27$ throughout their postbounce evolution have $a_{\mathrm{PBH}}^{*} \lesssim 0.9$. Similarly, PNSs with $T /|W| \lesssim 0.14-0.16$, the threshold for secular instability, have $a_{\mathrm{PBH}}^{*} \lesssim 0.6-0.7$. If low$T /|W|$ instabilities are effective at limiting $T /|W|$ in PNSs on short timescales, nascent $\mathrm{BH}$ spins may be limited to low values ( $a^{*} \lesssim 0.4$ for a $T /|W|$ instability threshold of $\sim 0.05$ ).

\subsubsection{Rotating Progenitors and the Connection to Long GRBs}

The rotation law of Equation (11) qualitatively follows the predicted angular velocity distribution in the inner $\sim 1-3 M_{\odot}$ of presupernova models evolved with rotation. However, Equation (11) asymptotes to constant specific angular momentum $j$ and cannot capture jumps and secular increase of $j$ in overlying mass shells (e.g., Heger et al. 2000).

Here, we consider three different supernova progenitors evolved with rotation that have the potential of forming BHs soon after bounce. Models E20 and E25 are rapidly rotating unmagnetized solar-metallicity presupernova models of a 20 and $25 M_{\odot}$ ZAMS stars from Heger et al. (2000). Model m35OC of Woosley \& Heger (2006) with $M_{\text {ZAMS }}=35 M_{\odot}$ has $10 \%$ solar-metallicity, reduced mass loss, and magnetic fields. These presupernova models have initial central angular velocities of $\sim 3.13, \sim 1.83$, and $\sim 1.98 \mathrm{rad} \mathrm{s}^{-1}$ and values of $\xi_{2.5}$ of $\sim 0.319$, $\sim 0.294$, and $\sim 0.456$ for the E20, E25, and m35OC models, respectively. Due to their moderate $\xi_{2.5}$, we perform collapse simulations of models E20 and E25 with the LS180 and LS220 EOS. Model m35OC is calculated with all four EOS. The progenitor characteristics are summarized in Tables 1 and 5.

For a given EOS, due to very similar $\xi_{2.5}$, the evolutions of E20 and E25 are alike. They form BHs in $\sim 1.1 \mathrm{~s}$ with the LS180 EOS and in $\sim 1.7 \mathrm{~s}$ with the LS220 EOS. Model E20 is more rapidly spinning. Its $T /|W|$ peaks at $\sim 0.18$ at bounce and settles down to a nearly constant value of $\sim 0.15$ throughout the postbounce evolution. Its $a_{\mathrm{PBH}}^{\star}$ is $\sim 0.7$ for both the LS180 and LS220 EOS. Model E25 reaches $T /|W| \sim 0.06$ at bounce and $\sim 0.065$ at $\mathrm{BH}$ formation with $a_{\mathrm{PBH}}^{\star} \sim 0.45$ for both EOS.

The core of the $\mathrm{m} 35 \mathrm{OC}$ model is sufficiently compact to form a BH soon after bounce if no explosion is launched (e.g., via magneto-rotational explosion; Dessart et al. 2008). The nascent $\mathrm{BH}$ forms at a time of $\sim 0.75 \mathrm{~s}, \sim 0.97 \mathrm{~s}, \sim 2.19 \mathrm{~s}$, and $\sim 1.91 \mathrm{~s}$ for the LS180, LS220, LS375, and HShen EOS, respectively. The initial gravitational (baryonic) $\mathrm{BH}$ masses are $\sim 2.22(\sim 2.33) M_{\odot}, \sim 2.29(\sim 2.43) M_{\odot}, \sim 2.69(\sim 3.01) M_{\odot}$, and $\sim 2.60(\sim 2.84) M_{\odot}$ for the LS180, LS220, LS375, and HShen EOS, respectively. The BHs are modestly rapidly spinning with $a_{\mathrm{PBH}}^{*}$ of $\sim 0.61, \sim 0.58, \sim 0.54$, and $\sim 0.55$ for the LS180, LS220, LS375, and HShen EOS, respectively. For all EOS, the PNS, during the accretion phase, has a modest $T /|W|$ of $\lesssim 0.12$.

Once a BH is formed, material from the stellar mantle will continue to accrete at high rates. Accretion will only be slowed once material with sufficiently high specific angular momentum reaches small radii and becomes centrifugally supported, forming an accretion disk. This is the crucial prerequisite for the collapsar scenario for long GRB central engines to work (Woosley 1993). Models E20 and E25 lost much of their initial mass and angular momentum during their evolution to the presupernova stage and, therefore, there is too little angular 


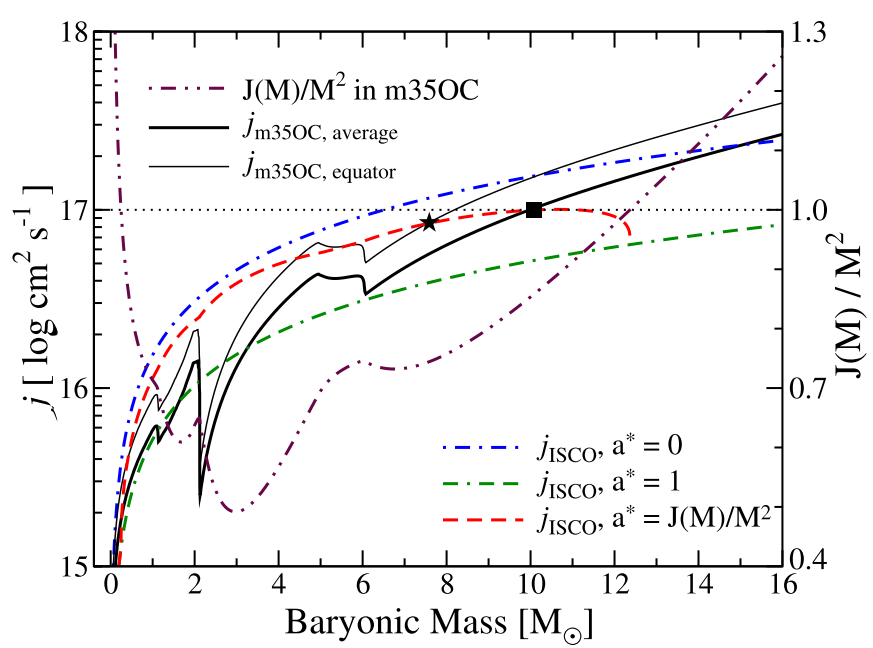

Figure 12. Specific angular momentum (solid, left ordinate) and dimensionless spin (dot-dot-dashed, right ordinate) for the GRB progenitor model m35OC from Woosley \& Heger (2006) as a function of enclosed baryonic mass. The thin solid line is the angular momentum at the equator; the thick solid line is the angleaveraged angular momentum. Also shown, dash-dotted, dash-dash-dotted, and dashed, is the specific angular momentum for a mass element at the innermost stable orbit assuming a background spacetime being Schwarzschild, maximally Kerr, and Kerr with $a^{*}=J(M) / M^{2}$, respectively. The horizontal line denotes $a^{*}=1$. ( $\star$ ) and $(\square)$ denote the mass coordinate where the equatorial and angle-average specific angular momenta exceed $j_{\text {ISCO }}$, respectively.

(A color version of this figure is available in the online journal.)

momentum in their outer regions to allow for a long-term accretion disk.

The situation is different for the m35OC progenitor. Its particular evolution prevented dramatic loss of mass and angular momentum while keeping its envelope radius small. In Figure 12, we show the specific angular momentum distribution of the m35OC progenitor as a function of enclosed baryonic mass. We also include graphs of the $j_{\text {ISCO }}$, the specific angular momentum required for a stable orbit at the innermost stable circular orbit (ISCO) of a Kerr hole with mass $M$ and spin $a^{\star}$ (Bardeen et al. 1972). Curves for $a^{*}=0$ (Schwarzschild), $a^{*}=1$ (maximally Kerr), and $a^{*}=J(M) / M^{2}$ are shown. We also plot the value of $a^{*}$ that a $\mathrm{BH}$ of baryonic mass $M$ formed from the m35OC progenitor would have. Figure 12 is independent of the detailed collapse evolution, assuming that no angular momentum is radiated by neutrinos and/or gravitational waves, or ejected. However, we note that due to emission of neutrinos before $\mathrm{BH}$ formation, the enclosed gravitational mass (entering into the calculation of $a^{*}$ ) will be smaller by up to $\sim 0.2-0.4 M_{\odot}$ than the baryonic mass given in the figure. This leads to slightly underpredicted values of $a^{*}$ for small $M$. Since relatively little energy is emitted in neutrinos after $\mathrm{BH}$ formation, the relative discrepancy between gravitational and baryonic mass decreases with growing $\mathrm{BH}$ mass.

Figure 12 can be interpreted as follows. If the CCSN mechanism fails to reenergize the shock and the PNS collapses to a $\mathrm{BH}$ of mass $M$, then its initial angular momentum $J$ and $\operatorname{spin} a^{\star}$ will be set by the enclosed angular momentum and gravitational mass. Initially, hyperaccretion will increase both $J$ and $M$, but $a^{\star}$ may increase or decrease, depending on the angular momentum of the accreted matter. Accretion will slow down and a disk will form once infalling material has specific angular momentum $j$ greater than $j_{\text {ISCO }}$. In model $\mathrm{m} 35 \mathrm{OC}$, this occurs between a $\mathrm{BH}$ mass coordinate of $\sim 7.6 M_{\odot}$ (for a mass element with equatorial $j$ ) and $\sim 10.1 M_{\odot}$ (for a mass element with angle-averaged $j)$. These points are marked in Figure 12 with a $(\star)$ and $(\mathbf{\square})$, respectively. Using accretion simulations with GR1D setup to include an inflow inner boundary condition we find that with the m35OC model, the accretion time for $7.6 M_{\odot}$ and $10.1 M_{\odot}$ is $\sim 10.2 \mathrm{~s}$ and $\sim 14.3 \mathrm{~s}$ from the onset of collapse, respectively. These times are roughly twice the free fall time, since material in outer regions in hydrostatic equilibrium for a sound travel time (Burrows 1986). Once the disk has formed, accretion will continue via processes that will transport angular momentum out and mass in. A collapsar central engine may begin its operation (Woosley 1993; MacFadyen \& Woosley 1999) with a central $\mathrm{BH}$ of $M \sim 8 M_{\odot}, a^{*} \sim 0.75$, and an ISCO radius of $\sim 40 \mathrm{~km}$.

\section{SUMMARY AND CONCLUSIONS}

We have performed an extensive study of $\mathrm{BH}$ formation in failing CCSNe with the open-source 1.5D GR code GR1D, making the simplifying assumptions of spherical symmetry and of a neutrino leakage scheme rather than full Boltzmann transport. We have performed more than 700 collapse simulations with over 100 unique progenitor models, probing systematically the many-dimensional parameter space that determines the outcome of stellar collapse in single massive stars. Specifically, we have studied and established the systematic dependence of CCSN failure and $\mathrm{BH}$ formation on progenitor compactness, precollapse rotational setup, neutrino heating efficiency, and nuclear EOS.

To first approximation, the evolution of any core collapse event proceeds as follows. Core collapse ensues in a given presupernova star, collapse is halted when the inner core of $\sim 0.5-0.7 M_{\odot}$ reaches nuclear density. A shock is formed, propagates outward initially in $M$ and $r$, but soon stalls. Assuming the CCSN mechanism, whatever its precise nature may be, fails, we can robustly predict the time it takes to $\mathrm{BH}$ formation for a given nuclear EOS (scaling with EOS stiffness) based on a single parameter, the progenitor bounce compactness $\xi_{2.5}$ $\left(t_{\mathrm{BH}} \propto \xi_{2.5^{-3 / 2}}\right)$. Using the same parameter, for a given EOS, we can predict the maximum mass of the PNS at collapse and its thermal enhancement (10\%-25\%) over the cold NS mass, due, as we have shown for the first time by detailed comparison with exact TOV solutions, primarily to thermal pressure support in the outer PNS core.

In an attempt to more quantitatively understand which stars explode and which do not, assuming the neutrino mechanism is responsible for the majority of CCSN explosions, we have turned the knobs on GR1D's neutrino heating scheme, experimentally, to first order, establishing the neutrino heating efficiency needed to explode a progenitor with given $\xi_{2.5}$. Neglecting the potentially highly relevant effects of multi-dimensional dynamics and assuming an EOS of intermediate stiffness (the LS220 EOS), we predict that progenitors with bounce compactness $\xi_{2.5} \gtrsim 0.45$ most likely form BHs without explosion. This prediction, in itself, without connection to ZAMS conditions through stellar evolution, is of limited utility. Using the whole set of progenitor data made available to us by stellar evolution groups, we attempt the former in Figure 13. We plot the mapping between ZAMS mass and outcome of core collapse, reduced to explosion or no explosion and $\mathrm{BH}$ formation, neglecting completely the possibility of $\mathrm{BH}$ formation due to fallback/cooling/phase transitions after a launched explosion. The case is clear cut at low metallicity where mass loss has negligible effect on the mapping between ZAMS conditions and core collapse outcome. Using a Salpeter initial mass function 
Outcome of Core Collapse (neglecting fallback, moderately-stiff EOS)

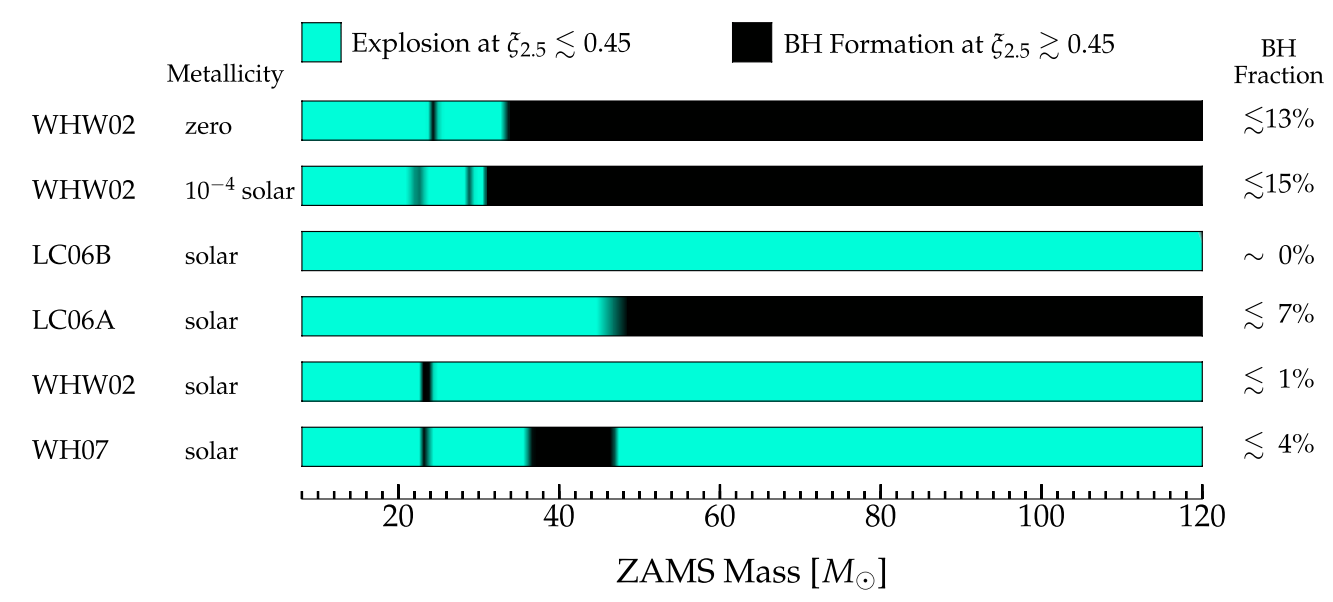

Figure 13. Outcome of core collapse as a function of ZAMS mass of single nonrotating massive stars, assuming that for moderately stiff nuclear EOS (e.g., LS180/ LS220), neutrino-driven explosions can be launched up to a bounce compactness $\xi_{2.5} \lesssim 0.45$ (cf. Section 4.5). Other potential explosion mechanisms are neglected. We consider only explosion and $\mathrm{BH}$ formation without explosion as outcomes and neglect other scenarios, including post-explosion $\mathrm{BH}$ formation via fallback accretion (Zhang et al. 2008; Dessart et al. 2010), cooling or nuclear phase transitions. Shown are results for a range of model sets and metallicities (see Section 3). Very low metallicity stars with ZAMS masses above $\sim 30 M_{\odot}$ robustly form a BH without explosion. At higher metallicity, uncertainties in the physics of mass loss (e.g., Smith et al. 2010) make robust predictions difficult. This is reflected in the rather dramatic disagreement of the four solar-metallicity progenitor model sets that we include. The "BH fractions" stated at the right edge of the plot denote the fraction of massive stars with $M \gtrsim 8 M_{\odot}$ that form BHs. They are obtained by convolution with a Salpeter IMF under the assumption that stars with $8 M_{\odot} \lesssim M \lesssim 14 M_{\odot}$ explode robustly.

(A color version of this figure is available in the online journal.)

(IMF; $\alpha=2.35, M_{\min }=8.0 M_{\odot}$, and $M_{\max }=150.0 M_{\odot}$ ) we estimate that $\sim 15 \%$ of all progenitors form BHs without explosion. At (around) solar metallicity, the precise way of prescribing mass loss in stellar evolution has tremendous consequences on the mapping between ZAMS mass and core collapse outcome. Depending on the particular mass-loss prescription, we predict a BH fraction of $0 \%-7 \%$ for solar-metallicity stars. This makes mass loss the single most important unknown parameter in connecting ZAMS conditions to core collapse outcome (in agreement with Smith et al. 2010).

Rapid rotation, which may be present in a significant subset of massive stars, generally increases the maximum PNS mass by centrifugal support and delays $\mathrm{BH}$ formation. Assuming (quite likely) uniform rotation of the PNS core, the increase in maximum PNS mass due to centrifugal support in the range of rotation rates explored is $\sim 5 \%-10 \%$. In the basic neutrino mechanism, rotation leads to a lower sum of $v_{e}$ and $\bar{v}_{e}$ luminosities and lower mean energies for all neutrino types. This is detrimental for explosion in 1.5D (and perhaps even in 2.5D) despite centrifugal support (Fryer \& Heger 2000; Ott et al. 2008). A larger fraction of massive stars may form BHs with (moderate) rotation than without. Left out of this picture are potential magnetohydrodynamics contributions to the explosion mechanism and energetics (cf. Burrows et al. 2007b).

Of particular interest to both formal relativity theory and astrophysics is the range of potential birth spins of BHs. Our results quite strikingly suggest that the rotation rate of the maximum-mass PNS and, hence, the spin of the nascent $\mathrm{BH}$, will be limited to values of $a^{\star}$ below $\lesssim 0.9$ by likely nonaxisymmetric dynamics. If true and confirmed by multidimensional simulations, 3D rotational instabilities may be a cosmic censor preventing naked singularities from forming in stellar collapse.

Rotation and the associated angular momentum are key ingredients in the collapsar scenario for GRBs (Woosley 1993). As part of this study, we have performed the first BH formation study with the m35OC GRB progenitor of Woosley \& Heger (2006). Using the LS220 EOS, we predict an initial BH mass of $\sim 2.29 M_{\odot}$ and $a^{\star}$ of $\sim 0.58$. Assuming that the GRB engine cannot operate until a Keplerian disk has formed, there will be a delay of $\sim 10 \mathrm{~s}$ between $\mathrm{BH}$ formation and GRB engine ignition at a BH mass of $\sim 8 M_{\odot}$ and $a^{\star} \sim 0.75$.

Finally, we re-emphasize that the goal of this study was not to yield accurate predictions about the outcome of core collapse in any individual progenitor. Rather, we have studied and established overall trends with progenitor parameters. We have made simplifications and approximations, and have omitted a broad range of potentially relevant physics. The most important of the latter may well be multi-dimensional dynamics and their effect on the CCSN explosion mechanism and on the associated failure rate of CCSNe.

Future work may be directed toward studying the systematics of $\mathrm{BH}$ formation in the post-explosion phase via fallback accretion, PNS cooling, or EOS phase transitions. Our current neutrino treatment must be upgraded for more quantitatively accurate simulations and neutrino signature predictions. Ultimately, multi-dimensional GR simulations of successful and failing CCSNe will be necessary to study the multi-dimensional dynamics left out here and for making truly robust predictions of the outcome of stellar collapse for any given set of initial conditions.

We acknowledge helpful discussions with and input from A. Burrows, P. Cerdá-Durán, L. Dessart, M. Duez, T. Fischer, J. Kaplan, J. Lattimer, C. Meakin, J. Murphy, F. Peng, S. Phinney, C. Reisswig, S. Scheidegger, N. Smith, E. Schnetter, K. Thorne, and S. Teukolsky. We thank S. Woosley and A. Heger for their recent presupernova models and A. Chieffi and M. Limongi for making available both of their presupernova model sets. The computations were performed at Caltech's Center for Advanced Computing Research on the cluster "Zwicky" funded through NSF grant no. PHY-0960291 and the Sherman Fairchild 
Foundation. Furthermore, computations were performed on Louisiana Optical Network Infrastructure computer systems under allocation loni_numrel05, on the NSF Teragrid under allocation TG-PHY100033, and on resources of the National Energy Research Scientific Computing Center, which is supported by the Office of Science of the U. S. Department of Energy under Contract No. DE-AC02-05CH11231. This research is supported in part by the National Science Foundation under grant nos. AST-0855535 and OCI-0905046. EOC is supported in part by a post-graduate fellowship from the Natural Sciences and Engineering Research Council of Canada (NSERC).

\section{REFERENCES}

Akiyama, S., Wheeler, J. C., Meier, D. L., \& Lichtenstadt, I. 2003, ApJ, 584 , 954

Arnett, W. D. 1966, Can. J. Phys., 44, 2553

Bardeen, J. M., Press, W. H., \& Teukolsky, S. A. 1972, ApJ, 178, 347

Baron, E., \& Cooperstein, J. 1990, ApJ, 353, 597

Baumgarte, T. W., Janka, H.-T., Keil, W., Shapiro, S. L., \& Teukolsky, S. A. 1996a, ApJ, 468, 823

Baumgarte, T. W., Shapiro, S. L., \& Teukolsky, S. A. 1996b, ApJ, 458, 680

Beacom, J. F., Boyd, R. N., \& Mezzacappa, A. 2001, Phys. Rev. D, 63, 073011

Belczynski, K., Kalogera, V., \& Bulik, T. 2002, ApJ, 572, 407

Bethe, H. A. 1990, Rev. Mod. Phys., 62, 801

Bethe, H. A., \& Wilson, J. R. 1985, ApJ, 295, 14

Bouret, J., Lanz, T., \& Hillier, D. J. 2005, A\&A, 438, 301

Buras, R., Janka, H.-T., Rampp, M., \& Kifonidis, K. 2006a, A\&A, 457, 281

Buras, R., Rampp, M., Janka, H.-T., \& Kifonidis, K. 2006b, A\&A, 447, 1049

Burrows, A. 1986, ApJ, 300, 488

Burrows, A. 1988, ApJ, 334, 891

Burrows, A., Dessart, L., \& Livne, E. 2007a, in AIP Conf. Ser. 937, Supernova 1987A: 20 Years After: Supernovae and Gamma-Ray Bursters, ed. S. Immler \& R. McCray (Melville, NY: AIP), 370

Burrows, A., Dessart, L., Livne, E., Ott, C. D., \& Murphy, J. 2007b, ApJ, 664, 416

Burrows, A., \& Lattimer, J. M. 1983, ApJ, 270, 735

Burrows, A., Livne, E., Dessart, L., Ott, C. D., \& Murphy, J. 2006, ApJ, 640, 878

Chandrasekhar, S. 1969, Ellipsoidal Figures of Equilibrium (revised edition 1987; New Haven, CT: Yale Univ. Press)

Chandrasekhar, S. 1970, ApJ, 161, 561

Colella, P., \& Woodward, P. R. 1984, J. Comput. Phys., 54, 174

Colgate, S. A., \& White, R. H. 1966, ApJ, 143, 626

Corvino, G., Rezzolla, L., Bernuzzi, S., De Pietri, R., \& Giacomazzo, B. 2010, Class. Quantum Grav., 27, 114104

de Jager, C., Nieuwenhuijzen, H., \& van der Hucht, K. A. 1988, A\&AS, 72, 259

Dessart, L., Burrows, A., Livne, E., \& Ott, C. D. 2008, ApJ, 673, L43

Dessart, L., Livne, E., \& Waldman, R. 2010, MNRAS, 408, 827

Dimmelmeier, H., Ott, C. D., Marek, A., \& Janka, H.-T. 2008, Phys. Rev. D, 78, 064056

Duez, M. D., Liu, Y. T., Shapiro, S. L., Shibata, M., \& Stephens, B. C. 2006, Phys. Rev. D, 73, 104015

Einfeldt, B. 1988, in Proc. 16th Int. Symp. on Shock Tubes and Waves, Aachen, Germany, 1987 July 26-31 (Weinheim, Germany: VCH Verlag), 671

Eldridge, J. J., \& Tout, C. A. 2004, MNRAS, 353, 87

Fernández, R., \& Thompson, C. 2009, ApJ, 703, 1464

Filippenko, A. V. 1997, Annu. Rev. Astron. Astrophys., 35, 309

Fischer, T., Whitehouse, S. C., Mezzacappa, A., Thielemann, F.-K., \& Liebendörfer, M. 2009, A\&A, 499, 1

Font, J. A. 2008, Living Rev. Rel., 11, 7

Friedman, J. L., \& Schutz, B. F. 1978, ApJ, 222, 281

Fryer, C. L. 1999, ApJ, 522, 413

Fryer, C. L., \& Heger, A. 2000, ApJ, 541, 1033

Fryer, C. L., \& Kalogera, V. 2001, ApJ, 554, 548

Fullerton, A. W., Massa, D. L., \& Prinja, R. K. 2006, ApJ, 637, 1025

Gourgoulhon, E. 1991, A\&A, 252, 651

Heger, A., Fryer, C. L., Woosley, S. E., Langer, N., \& Hartmann, D. H. 2003, ApJ, 591,288

Heger, A., Langer, N., \& Woosley, S. E. 2000, ApJ, 528, 368

Hyman, J. M. 1976, The Method of Lines Solution of Partial Differential Equations, Technical Report, ERDA Mathematics and Computing Laboratory, Courant Institute of Mathematical Sciences, New York University
Ishizuka, C., Ohnishi, A., Tsubakihara, K., Sumiyoshi, K., \& Yamada, S. 2008, J. Phys. G: Nucl. Phys., 35, 085201

Janka, H.-T. 2001, A\&A, 368, 527

Janka, H.-T., Langanke, K., Marek, A., Martínez-Pinedo, G., \& Müller, B. 2007, Phys. Rep., 442, 38

Kitaura, F. S., Janka, H.-T., \& Hillebrandt, W. 2006, A\&A, 450, 345

Kochanek, C. S., Beacom, J. F., Kistler, M. D., Prieto, J. L., Stanek, K. Z., Thompson, T. A., \& Yüksel, H. 2008, ApJ, 684, 1336

Lai, D., \& Shapiro, S. L. 1995, ApJ, 442, 259

Langer, N. 1989, A\&A, 220, 135

Lattimer, J. M., \& Swesty, F. D. 1991, Nucl. Phys. A, 535, 331

Liebendörfer, M. 2005, ApJ, 633, 1042

Liebendörfer, M., Messer, O. E. B., Mezzacappa, A., Bruenn, S. W., Cardall, C. Y., \& Thielemann, F.-K. 2004, ApJS, 150, 263

Liebendörfer, M., Rampp, M., Janka, H.-T., \& Mezzacappa, A. 2005, ApJ, 620, 840

Limongi, M., \& Chieffi, A. 2006, ApJ, 647, 483

Limongi, M., \& Chieffi, A. 2009, Mem. Soc. Astron. Ital., 80, 151

MacFadyen, A. I., \& Woosley, S. E. 1999, ApJ, 524, 262

Marek, A., \& Janka, H.-T. 2009, ApJ, 694, 664

Meynet, G., \& Maeder, A. 2003, A\&A, 404, 975

Murphy, J. W., \& Burrows, A. 2008, ApJ, 688, 1159

Nakazato, K., Sumiyoshi, K., \& Yamada, S. 2010, ApJ, 721, 1284

Nieuwenhuijzen, H., \& de Jager, C. 1990, A\&A, 231, 134

Nordhaus, J., Burrows, A., Almgren, A., \& Bell, J. 2010, ApJ, 720, 694

Nugis, T., \& Lamers, H. J. G. L. M. 2000, A\&A, 360, 227

O'Connor, E., \& Ott, C. D. 2010, Class. Quantum Grav., 27, 114103

Oppenheimer, J. R., \& Volkoff, G. M. 1939, Phys. Rev., 55, 374

Ott, C. D. 2009, Class. Quantum Grav., 26, 063001

Ott, C. D., Burrows, A., Dessart, L., \& Livne, E. 2008, ApJ, 685, 1069

Ott, C. D., Burrows, A., Thompson, T. A., Livne, E., \& Walder, R. 2006, ApJS, 164,130

Ott, C. D., Dimmelmeier, H., Marek, A., Janka, H.-T., Hawke, I., Zink, B., \& Schnetter, E. 2007, Phys. Rev. Lett., 98, 261101

Ott, C. D., \& O'Connor, E. 2010, in AIP Conf. Ser. 1269, 10th Int. Symp. on Origin of Matter and Evolution of Galaxies: OMEG2010, ed. I. Tanihara et al. (Melville, NY: AIP), 166

Penrose, R. 1969, Nuovo Cimento Riv. Ser., 1, 252

Petrich, L. I., Shapiro, S. L., \& Teukolsky, S. A. 1986, Phys. Rev. D, 33, 2100

Pons, J. A., Reddy, S., Prakash, M., Lattimer, J. M., \& Miralles, J. A. 1999, ApJ, 513, 780

Puls, J., Markova, N., Scuderi, S., Stanghellini, C., Taranova, O. G., Burnley, A. W., \& Howarth, I. D. 2006, A\&A, 454, 625

Romero, J. V., Ibanez, J. M., Marti, J. M., \& Miralles, J. A. 1996, ApJ, 462, 839

Rosswog, S., \& Liebendörfer, M. 2003, MNRAS, 342, 673

Ruffert, M., Janka, H.-T., \& Schäfer, G. 1996, A\&A, 311, 532

Sagert, I., Fischer, T., Hempel, M., Pagliara, G., Schaffner-Bielich, J., Mezzacappa, A., Thielemann, F., \& Liebendörfer, M. 2009, Phys. Rev. Lett., 102,081101

Saijo, M., Baumgarte, T. W., \& Shapiro, S. L. 2003, ApJ, 595, 352

Scheidegger, S., Fischer, T., Whitehouse, S. C., \& Liebendörfer, M. 2008, A\&A, 490, 231

Sekiguchi, Y.-I., \& Shibata, M. 2004, Phys. Rev. D, 70, 084005

Sekiguchi, Y.-I., \& Shibata, M. 2005, Phys. Rev. D, 71, 084013

Sekiguchi, Y.-I., \& Shibata, M. 2010, ApJ, submitted (arXiv:1009.5303)

Shen, H., Toki, H., Oyamatsu, K., \& Sumiyoshi, K. 1998a, Nucl. Phys. A, 637, 435

Shen, H., Toki, H., Oyamatsu, K., \& Sumiyoshi, K. 1998b, Prog. Theor. Phys., 100,1013

Shibata, M., \& Sekiguchi, Y.-I. 2005, Phys. Rev. D, 71, 024014

Smartt, S. J., Eldridge, J. J., Crockett, R. M., \& Maund, J. R. 2009, MNRAS, 395, 1409

Smith, N. 2008, in IAU Symp. 250, Massive Stars as Cosmic Engines, ed. F. Bresolin, P. A. Crowther, \& J. Puls (Cambridge: Cambridge Univ. Press), 193

Smith, N., Li, W., Filippenko, A. V., \& Chornock, R. 2010, ApJ, submitted (arXiv:1006.3899)

Stergioulas, N. 2003, Living Rev. Rel., 6, 3

Sumiyoshi, K., Ishizuka, C., Ohnishi, A., Yamada, S., \& Suzuki, H. 2009, ApJ, 690, L43

Sumiyoshi, K., Yamada, S., \& Suzuki, H. 2007, ApJ, 667, 382

Sumiyoshi, K., Yamada, S., \& Suzuki, H. 2008, ApJ, 688, 1176

Thompson, T. A., Burrows, A., \& Pinto, P. A. 2003, ApJ, 592, 434

Thompson, T. A., Quataert, E., \& Burrows, A. 2005, ApJ, 620, 861

Timmes, F. X., Woosley, S. E., \& Weaver, T. A. 1996, ApJ, 457, 834 
van Riper, K. A., \& Arnett, W. D. 1978, ApJ, 225, L129

Vink, J. S., \& de Koter, A. 2005, A\&A, 442, 587

Vink, J. S., de Koter, A., \& Lamers, H. J. G. L. M. 2000, A\&A, 362, 295

Vink, J. S., de Koter, A., \& Lamers, H. J. G. L. M. 2001, A\&A, 369, 574

Wellstein, S., \& Langer, N. 1999, A\&A, 350, 148

Wilson, J. R. 1971, ApJ, 163, 209
Woosley, S. E. 1993, ApJ, 405, 273

Woosley, S. E., \& Heger, A. 2006, ApJ, 637, 914

Woosley, S. E., \& Heger, A. 2007, Phys. Rep., 442, 269

Woosley, S. E., Heger, A., \& Weaver, T. A. 2002, Rev. Mod. Phys., 74, 1015

Woosley, S. E., \& Weaver, T. A. 1995, ApJS, 101, 181

Zhang, W., Woosley, S. E., \& Heger, A. 2008, ApJ, 679, 639 